NBER WORKING PAPER SERIES

\title{
AN ANALYSIS OF THE MEMPHIS NURSE-FAMILY PARTNERSHIP PROGRAM
}

\author{
James J. Heckman \\ Margaret L. Holland \\ Kevin K. Makino \\ Rodrigo Pinto \\ Maria Rosales-Rueda \\ Working Paper 23610 \\ http://www.nber.org/papers/w23610
}

\author{
NATIONAL BUREAU OF ECONOMIC RESEARCH \\ 1050 Massachusetts Avenue \\ Cambridge, MA 02138 \\ July 2017
}

We are very grateful to Professor David Olds, founder of the NFP, and his team for generously sharing the data and source materials from the NFP Memphis Randomize Control Trial. Years of collaboration and productive discussions with him have made this study possible. We thank Terrance Oey and Willem van Vliet for superb research assistance. We are grateful to Juan Pantano, Sylvi Kuperman, Jorge Luis Garcia, Maryclare Griffin, Andres Hojman, Yu Kyung Koh, Cullen Roberts, Karl Schulze, Naoko Takeda, and Joyce Zhu for helpful comments. An early version of this paper was presented at seminars at the University of Chicago and the Association for Public Policy Analysis and Management 2012 Fall conference. This research was supported by NICHD R37HD065072 and NICHD R01HD054702, and previous support of the Pritzker Children's Initiative. A portion of Holland's time on this project was also supported by an NRSA Institutional Research Training Grant from the Health Resources and Services Administration (T32 PE12002), awarded to the Department of Pediatrics at the University of Rochester. The views expressed in this paper are solely those of the authors and do not necessarily represent the official views of the National Institutes of Health. The views expressed herein are those of the authors and do not necessarily reflect the views of the National Bureau of Economic Research.

NBER working papers are circulated for discussion and comment purposes. They have not been peer-reviewed or been subject to the review by the NBER Board of Directors that accompanies official NBER publications.

(C) 2017 by James J. Heckman, Margaret L. Holland, Kevin K. Makino, Rodrigo Pinto, and Maria Rosales-Rueda. All rights reserved. Short sections of text, not to exceed two paragraphs, may be quoted without explicit permission provided that full credit, including $(\odot)$ notice, is given to the source. 
An Analysis of the Memphis Nurse-Family Partnership Program

James J. Heckman, Margaret L. Holland, Kevin K. Makino, Rodrigo Pinto, and Maria Rosales-Rueda NBER Working Paper No. 23610

July 2017

JEL No. C5,H5,I1

\begin{abstract}
$\underline{\text { ABSTRACT }}$
This paper evaluates a randomized controlled trial of the Nurse-Family Partnership (NFP) program conducted in Memphis, TN in 1990. NFP offers home visits conducted by nurses for disadvantaged first-time mothers during pregnancy and early childhood. We test NFP treatment effects using permutation-based inference that accounts for the NFP randomization protocol. Our methodology is valid for small samples and corrects for multiple-hypothesis testing. We also analyze the underlying mechanisms generating these treatment effects. We decompose NFP treatment effects into components associated with the intervention-enhanced parenting and early childhood skills. The NFP improves home investments, parenting attitudes and mental health for mothers of infants at age 2. At age 6, the NFP boosts cognitive skills for both genders and socioemotional skills for females. These treatment effects are explained by program-induced improvements in maternal traits and early-life family investments. At age 12, the treatment effects for males (but not for females) persist in the form of enhanced achievement test scores. Treatment effects are largely explained by enhanced cognitive skills at age 6. Our evidence of pronounced gender differences in response to early childhood interventions contributes to a growing literature on this topic.
\end{abstract}

James J. Heckman

Department of Economics

The University of Chicago

1126 E. 59th Street

Chicago, IL 60637

and IZA

and also NBER

jjh@uchicago.edu

Margaret L. Holland

Yale School of Nursing

400 West Campus Drive

Orange CT 06477

margaret.holland@yale.edu

Kevin K. Makino

John A. Burns School of Medicine

University of Hawaii

1319 Puhanou Street

Honolulu, HI 96826

kkmakino@hawaii.edu
Rodrigo Pinto

University of California, Los Angeles

Department of Economics

8283 Bunche Hall

Los Angeles, CA 90095

rodrig@econ.ucla.edu

Maria Rosales-Rueda

University of California - Irvine

School of Education

Irvine CA 92697-5500

mfrosale@uci.edu

Supplementary materials available at http://www.nber.org/papers/w23610:

- data appendix 


\section{Contents}

1 Introduction $\quad 3$

2 Experimental Design and Background $\quad 6$

3 Inference $\quad 12$

4 Estimated Treatment Effects $\quad 14$

5 Understanding the Sources of NFP Treatment Effects 26

5.1 Empirical Strategy to Decompose Treatment Effects . . . . . . . . . . . . . . 28

6 Decomposing Treatment Effects 30

6.1 NFP Effects on Skills at Ages 2 and 6 . . . . . . . . . . . . 30

6.2 Decomposition of Treatment Effects at Age 6 . . . . . . . . . . . . . . 31

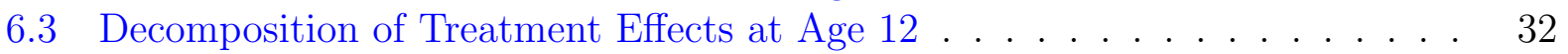

7 Summary and Conclusion $\quad 43$ 


\section{Introduction}

Home visiting programs are widely used to improve the well-being of disadvantaged pregnant women and their children (Currie and Rossin-Slater, 2015; Howard and Brooks-Gunn, 2009a). In 2010, the United States expanded federal funding for the establishment of the Maternal, Infant, and Early Childhood Home Visiting Program (MIECHV Program) to support state implementation of early childhood home visiting programs. At present, funding for MIECHV Program has been been reauthorized, with bipartisan support for the program. Between 2012-2015, the MIECHV program served more than 300,000 disadvantaged families and children in the US. ${ }^{1}$

The Nurse-Family Partnership (NFP) is widely considered the primary MIECHV Program among evidence-based home visiting models. The NFP offers prenatal and early childhood care to disadvantaged, unmarried, first-time mothers. Treatment consists of home visits starting during pregnancy and lasting until two years after birth. The NFP home visits are conducted by professional registered nurses with at least of a Bachelor of Science degree in nursing. They have formal training and follow a detailed curriculum of activities specific to the different stages of pregnancy and child development (Currie and Rossin-Slater, 2015). The NFP aims to improve the long-term success of disadvantaged children. It operates by promoting healthy maternal behaviors and by fostering parenting skills. Prior to being implemented at scale, three randomized controlled trials (RCTs) were performed to assess the impact of the NFP in different populations. ${ }^{2}$

Today, NFP surrogates operate in 43 states across the U.S. and have provided service for more than 200, 000 families since 1996. A version of NFP is currently operating in the U.K. and Germany (Robling et al., 2016; Sandner et al., 2017).

Most of the NFP literature focuses on three social experiments targeting poor high-risk

\footnotetext{
${ }^{1}$ Between 2010-2014, the MIECHV Program provided $\$ 1.5$ billion to states for implementing home visiting programs. In 2015, a two year extension was approved to continue supporting these programs until 2017. The program is currently up for renewal.

${ }^{2}$ The Memphis randomization protocol is presented in Appendix A. A summary of previous experiments is presented in Appendix B. Appendix K summarizes the findings of previous studies.
} 
pregnant females. The first NFP trial occurred in Elmira (1978) and its sample consists of primary non-Hispanic white mothers. The second trial took place in Memphis (1990) and targeted mainly non-Hispanic blacks. A third intervention occurred in Denver (1994) and overrepresented Hispanic participants. This paper uses data from the Memphis randomized control trial to examine NFP impacts through age 12, and to investigate the mechanisms producing the treatment effects.

There are several previous evaluations of the NFP that examine its effects on a range of domains, including maternal and child health, child development, parenting, and family economic self-sufficiency (Avellar et al., 2011; Daro, 2006; Olds, 2012). ${ }^{3}$ However, the literature does not account for features of the randomization protocol (such as stratification), does not control for multiple-hypothesis testing (i.e., control for "cherry picking") and does not analyze the underlying channels generating treatment effects. Our analysis presents a rigorous examination that addresses these issues. The Memphis randomized control trial is the only NFP intervention that collects detailed item-level data necessary to analyze different channels of influence. In particular, the Memphis trial gathered rich information on mother and child well-being from pregnancy up to age 12. It includes information on maternal behavior, home environments, parenting, children's cognitive and socio-emotional skills measured at ages 2 and 6, and middle childhood outcomes such as achievement and behavior problems, measured at age 12 .

We contribute to the existing NFP literature in three ways. First, our paper is the first to conduct inference that accounts for the features of the NFP randomization protocol and addresses the problem of cherry picking. We use permutation-based inference and implement multiple-hypothesis testing adjustments using the step down procedure of Romano and Wolf (2005). We show that most of the findings from previous papers survive permutation testing. However, many fewer treatment effects survive corrections for multiple-hypothesis testing.

Second, our paper is the first to analyze the effects of the NFP by gender, following

\footnotetext{
${ }^{3}$ Our results broadly corroborate the findings of Olds (2012) while producing new insights on the NFP intervention.
} 
the previous literature that has found gender differences in early-childhood program effects (see Elango et al., 2016; García et al., 2017b; Heckman et al., 2010 and García, Heckman, and Ziff, 2017). Third, this is the first paper that conducts a mediation analysis of NFP treatment effects. Following the methodology developed by Heckman et al. (2013), we decompose statistically significant treatment effects into interpretable components associated with program-induced changes in children's early skills and parental investments. We estimate the channels underlying treatment effects at age 6 (using maternal skills and investment effects at the end of the program as mediators) and at age 12 (using the program effects at age 6 as mediators).

We find statistically significant treatment effects of NFP on home environments, parenting attitudes, and maternal mental health for parents of both boys and girls at age 2 . At age 6, the program improved cognitive skills for both boys and girls, while it enhanced early socio-emotional skills for girls. These treatment effects arise from program-induced improvements in maternal traits and early-life family investments at age 2 . The treatment effects for males persist through age 12. Treated males outperform controls on a range of cognitive achievement scores. We find that 40\%-60\% of male treatment effects at age 12 can be explained by enhanced cognitive skills measured at age 6 . The program has much weaker long-term effects for girls. This finding is consistent with the evidence in Elango et al. (2016) and García et al. (2017a). This paper contributes to the literature on the positive influences of early childhood interventions. We show that parenting matters. Specifically, we find that program-induced improvements in maternal mental health (reduction in maternal anxiety and improvement on mastery/self-control) and program-induced enhancement of parenting skills are strong channels underlying program effects on children's skills. We also present evidence that the NFP had long-lasting effects on cognitive skills for boys, which contrast with the limited evidence on the persistent effects of preschool interventions on cognition. One possible explanation for the long-term effects of NFP on cognitive skills for boys is the health effects of the home visiting program on birth weight. This explains a large share 
of the age 12 program impact on cognition. This finding suggests that early childhood interventions that start before birth have the potential to affect cognitive skill formation for which sensitive periods occur in utero and in the first years of life (see Nelson, 2012).

The remainder of this paper is organized as follows. Section 2 provides information on the experimental design, background variables and measured outcomes of the NFP Memphis trial. Section 3 explains our inferential approach. Section 4 presents our empirical results. Section 5 describes the methodology used to decompose treatment effects. Section 6 presents our mediation analysis. Section 7 concludes.

\section{Experimental Design and Background}

The NFP Memphis trial was initiated in 1990. The main goals of NFP are: (1) improvement of maternal and fetal health during pregnancy; (2) development of parenting skills; and (3) planning of social and economic aspects of maternal life through counseling services (Olds, 2002; Olds et al., 1997).

Visits started during pregnancy and continued until the newborn reached the age of 2 . During these visits, nurses encouraged mothers to adopt a healthy diet and eliminate the use of tobacco, alcohol, and illegal drugs. Nurses also taught mothers to recognize complications in pregnancy and to administer adequate prenatal care. After delivery, nurses promoted infant health care and good parenting skills. Mothers were instructed on how to interact with their children in order to foster emotional and cognitive development. NFP further assisted mothers in the process of establishing life goals related to work, education and future pregnancies.

Table 1 summarizes the main elements of NFP. We discuss its features in detail. We compare the designs of the different NFP studies in Appendix B. 


\section{Table 1: Nurse-Family Partnership Intervention: Memphis Trial}

\begin{tabular}{|c|c|}
\hline Intervention Goals & $\begin{array}{l}\text { Healthy prenatal behaviors } \\
\text { Parenting skills } \\
\text { Life-planning strategies }\end{array}$ \\
\hline Target Population & $\begin{array}{l}\text { Low-income first-time mothers } \\
\text { During pregnancy to two years of age }\end{array}$ \\
\hline Eligibility Criteria & $\begin{array}{l}\text { Biological Criteria: all mothers must comply with the following criteria } \\
\text { 1. Less than } 29 \text { weeks of pregnancy } \\
\text { 2. No previous live births } \\
\text { 3. No specific chronic illness affecting fetus } \\
\text { Socio-Economic Criteria: at least two of the following indicators } \\
\text { 1. Unmarried } \\
\text { 2. Less than } 12 \text { years of education } \\
\text { 3. Unemployed }\end{array}$ \\
\hline Sociodemographic & $\begin{array}{l}\text { Race: } 92 \% \text { African-American } \\
\text { Marital Status: } 97 \% \text { unmarried } \\
\text { Income: } 85 \% \text { low-income } \\
\text { Age: } 64 \% \text { under } 18\end{array}$ \\
\hline NFP Groups & $\begin{array}{l}\text { Group } 1 \text { (Sample 166) } \\
\text { Free transportation to and from appointments (not followed after birth) } \\
\text { Group } 2 \text { (Sample } 514 \text { ) } \\
\text { Free transportation and developmental screening at ages } 6,12,24 \text { months } \\
\text { Group } 3 \text { (Sample } 230 \text { ) } \\
\text { Home visits by nurse during pregnancy plus Group } 2 \text { benefits (not followed after birth) } \\
\text { Group } 4 \text { (Sample 228) } \\
\text { Regular home visits during child's infancy plus all the Group } 3 \text { benefits }\end{array}$ \\
\hline Treatment Groups & In our analysis, Group 2 is our "control group" and Group 4 is our "treatment group." \\
\hline Frequency of Visits & $\begin{array}{l}\text { Weekly for the first month after enrollment } \\
\text { Bi-monthly from second month after enrollment until birth } \\
\text { Weekly for the first } 6 \text { weeks after birth } \\
\text { Bi-monthly from seventh week after birth until child is } 20 \text { months } \\
\text { Monthly from } 21 \text { months of age until child is } 24 \text { months }\end{array}$ \\
\hline Dosage & $\begin{array}{l}\text { Average number of visits during pregnancy: } 7 \text { visits } \\
\text { Average number of visits during infancy (0-24 months): } 26 \text { visits }\end{array}$ \\
\hline
\end{tabular}

Notes: Basic information on the NFP Memphis Trial. See Kitzman et al. (1997) for a detailed description. 


\section{Eligibility}

The NFP Memphis trial recruited pregnant women from June 1, 1990 to August 31, 1991 through the Memphis-Shelby County Tennessee Health Department. Eligible mothers satisfied the following biological criteria: (1) less than 29 weeks of pregnancy; (2) no previous live birth; and (3) no chronic illnesses that could contribute to fetal-growth retardation or preterm delivery. They also satisfied two or more of the following socio-economic criteria: (1) unmarried; (2) less than 12 years of education; and (3) unemployed.

\section{Sample}

The total sample includes 1,290 invited participants and 1,138 mothers effectively enrolled. The majority of the participants were African-American (92\%), unmarried (97\%), low income (95\%), and under 18 years old (64\%). Mothers who participated in the program were more likely to be younger and African-American and less likely to have completed high school compared to mothers who refused to participate (Kitzman et al., 1997).

\section{Treatment}

The mothers who agreed to participate in the trial were randomized into four different groups that differed by treatment:

- Group 1: Round-trip transportation from their homes to prenatal session appointments (sample size: 166).

- Group 2: Developmental screening and referral services when their babies were age 6, 12, and 24 months, in addition to the benefits of Group 1 (sample size: 514). ${ }^{4}$

- Group 3: Nurse visits during pregnancy, one visit when in the hospital and one visit at home after childbirth, in addition to the benefits of Group 2 (sample size: 230).

- Group 4: Nurse home visits during pregnancy and until the child's second birthday, in addition to the benefits of Group 2 (sample size: 228).

\footnotetext{
${ }^{4}$ An infant specialist hired by the research project screened the children for sensory and developmental problems and referred those with suspected problems to other specialists for further evaluation and treatment.
} 
There are no available data on the participants of Groups 1 and 3 after the child's birth, as those participants were not followed. Our paper only uses data on the participants who were originally assigned to Groups 2 or 4 . These treatment groups comply with the selection of treatment groups used in previous evaluations other NFP trials. (See Appendix B.) Henceforth, we term Group 4 as the treatment group and Group 2 as control group. It is worth noting that Group 2 received some benefits in the form of developmental screening.

Table 2 presents a summary of selected baseline characteristics for the treatment and control groups by gender of the child. The table shows that pre-program characteristics are fairly balanced across treatment groups. Some exceptions are that treated mothers have lower income, lower likelihood of being in school, lower employment status of the household head, and higher grandmother support compared to mothers in the control group. ${ }^{5}$

\section{Randomization protocol}

The NFP randomization was performed within strata defined by 5 characteristics: (1) maternal race (African-American vs non-African-American); (2) maternal age ( $<17,17-18$, $>18$ years ); (3) gestational age at enrollment ( $<20, \geq 20$ weeks); (4) employment status of the head of household; and (5) geographic region of residence. ${ }^{6}$ Appendix A provides a detailed description of the randomization protocol.

\section{Attrition}

Post-randomization dropout was rare during the program period: four women in both treatment and control groups refused further participation in the intervention after randomization. There were 487 live births in the control group (out of 514 pregnant mothers) and 214 live births in the treatment group (out of 228 pregnant mothers). There were 27 miscarriages in the control group and 14 in the treatment group. Table E.1 in Appendix E shows the percentage of available data (retention rates) by gender and age of follow-up interviews. The differences in attrition by group are not statistically significant. When the children were

\footnotetext{
${ }^{5}$ In addition, control mothers of male children were slightly taller than their treatment counterparts.

${ }^{6}$ The regions are: Inner City, Bisson, Cawthon and Hollywood.
} 


\section{Table 2: Descriptive Statistics of NFP Baseline Characteristics}

\begin{tabular}{|c|c|c|c|c|c|c|c|c|c|c|}
\hline Background Characteristics & $\begin{array}{c}\text { Control } \\
\text { Mean }\end{array}$ & $\begin{array}{l}\text { Fem } \\
\text { Control } \\
\text { Std.Dev. }\end{array}$ & $\begin{array}{r}\text { ale San } \\
\text { Treat- } \\
\text { ment } \\
\text { Mean }\end{array}$ & $\begin{array}{l}\text { nple } \\
\text { Treat- } \\
\text { ment } \\
\text { Std.Dev. }\end{array}$ & $p$-val & $\begin{array}{r}\text { Control } \\
\text { Mean }\end{array}$ & $\begin{array}{l}\text { Ma } \\
\text { Control } \\
\text { Std.Dev. }\end{array}$ & $\begin{array}{r}\text { le Sam } \\
\text { Treat- } \\
\text { ment } \\
\text { Mean }\end{array}$ & $\begin{array}{l}\text { ple } \\
\text { Treat- } \\
\text { ment } \\
\text { Std.Dev. }\end{array}$ & $p$-val \\
\hline $\begin{array}{r}\text { Maternal Race (White) } \\
\text { Marital Status (Married) } \\
\text { Maternal Age } \\
\text { Years of Education } \\
\text { Mother in School } \\
\text { Head of Household Is Employed } \\
\% \text { of Census Tract Below Poverty } \\
\text { Household Density } \\
\text { Total Household Income (Past } 6 \text { Months) }\end{array}$ & $\begin{array}{l}0.086 \\
0.012 \\
18.32 \\
10.38 \\
0.541 \\
0.607 \\
32.53 \\
0.944\end{array}$ & $\begin{array}{l}0.281 \\
0.110 \\
3.290 \\
1.833 \\
0.499 \\
0.490 \\
20.23 \\
0.490\end{array}$ & $\begin{array}{l}0.101 \\
0.009 \\
18.27 \\
10.15 \\
0.596 \\
0.495 \\
36.29 \\
1.053\end{array}$ & $\begin{array}{r}0.303 \\
0.096 \\
3.531 \\
2.040 \\
0.493 \\
0.502 \\
21.820 \\
0.652\end{array}$ & $\begin{array}{r}0.656 \\
0.791 \\
0.877 \\
0.300 \\
0.333 \\
\mathbf{0 . 0 5 4} \\
0.128 \\
0.123\end{array}$ & $\begin{array}{r}0.076 \\
0.025 \\
17.95 \\
10.25 \\
0.630 \\
0.517 \\
36.047 \\
0.923\end{array}$ & $\begin{array}{l}0.265 \\
0.157 \\
3.130 \\
1.919 \\
0.484 \\
0.501 \\
22.10 \\
0.490\end{array}$ & $\begin{array}{r}0.112 \\
0.019 \\
17.96 \\
10.112 \\
0.533 \\
0.519 \\
34.25 \\
0.983\end{array}$ & $\begin{array}{l}0.317 \\
0.136 \\
2.962 \\
1.997 \\
0.501 \\
0.502 \\
18.32 \\
0.446\end{array}$ & $\begin{array}{r}0.300 \\
0.696 \\
0.970 \\
0.543 \\
\mathbf{0 . 0 9 3} \\
0.972 \\
0.431 \\
0.264\end{array}$ \\
\hline $\begin{array}{r}\text { Less Than } \$ 3000 \\
\$ 3000-\$ 6999 \\
\$ 7000-\$ 10999 \\
\text { Greater Than } \$ 11000 \\
\text { Income, No Response }\end{array}$ & $\begin{array}{l}0.278 \\
0.237 \\
0.216 \\
0.180 \\
0.090\end{array}$ & $\begin{array}{l}0.449 \\
0.426 \\
0.413 \\
0.385 \\
0.286\end{array}$ & $\begin{array}{l}0.358 \\
0.220 \\
0.229 \\
0.083 \\
0.110\end{array}$ & $\begin{array}{l}0.482 \\
0.416 \\
0.422 \\
0.277 \\
0.314\end{array}$ & $\begin{array}{r}0.141 \\
0.732 \\
0.788 \\
\mathbf{0 . 0 0 8} \\
0.565\end{array}$ & $\begin{array}{l}0.286 \\
0.248 \\
0.235 \\
0.134 \\
0.097\end{array}$ & $\begin{array}{l}0.453 \\
0.433 \\
0.425 \\
0.342 \\
0.296\end{array}$ & $\begin{array}{l}0.355 \\
0.224 \\
0.178 \\
0.178 \\
0.065\end{array}$ & $\begin{array}{l}0.481 \\
0.419 \\
0.384 \\
0.384 \\
0.248\end{array}$ & $\begin{array}{l}0.208 \\
0.632 \\
0.213 \\
0.320 \\
0.311\end{array}$ \\
\hline $\begin{array}{r}\text { Inner City } \\
\text { Bisson } \\
\text { Cawthon } \\
\text { Hollywood }\end{array}$ & $\begin{array}{l}0.282 \\
0.171 \\
0.229 \\
0.318\end{array}$ & $\begin{array}{l}0.451 \\
0.378 \\
0.421 \\
0.467\end{array}$ & $\begin{array}{l}0.284 \\
0.229 \\
0.174 \\
0.312\end{array}$ & $\begin{array}{l}0.453 \\
0.422 \\
0.381 \\
0.465\end{array}$ & $\begin{array}{l}0.958 \\
0.220 \\
0.233 \\
0.905\end{array}$ & $\begin{array}{l}0.298 \\
0.210 \\
0.185 \\
0.307\end{array}$ & $\begin{array}{l}0.458 \\
0.408 \\
0.389 \\
0.462\end{array}$ & $\begin{array}{l}0.280 \\
0.206 \\
0.224 \\
0.290\end{array}$ & $\begin{array}{l}0.451 \\
0.406 \\
0.419 \\
0.456\end{array}$ & $\begin{array}{l}0.734 \\
0.925 \\
0.410 \\
0.750\end{array}$ \\
\hline $\begin{array}{r}\text { Maternal IQ (Shipley) } \\
\text { Maternal Bavolek Score } \\
\text { Maternal Mental Health } \\
\text { Self-Efficacy } \\
\text { Maternal Mastery } \\
\text { Maternal Psychological Resources } \\
\text { Maternal Health Characteristics }\end{array}$ & $\begin{array}{c}96.43 \\
99.77 \\
99.73 \\
100.71 \\
100.13 \\
100.15\end{array}$ & $\begin{array}{r}10.30 \\
7.63 \\
10.12 \\
9.836 \\
10.202 \\
9.903\end{array}$ & $\begin{array}{r}96.51 \\
100.93 \\
99.12 \\
100.55 \\
99.10 \\
99.51\end{array}$ & $\begin{array}{r}10.46 \\
8.76 \\
10.53 \\
9.28 \\
10.11 \\
10.43\end{array}$ & $\begin{array}{l}0.949 \\
0.233 \\
0.610 \\
0.889 \\
0.375 \\
0.586\end{array}$ & $\begin{array}{r}96.32 \\
99.57 \\
100.66 \\
99.44 \\
100.32 \\
100.15\end{array}$ & $\begin{array}{r}10.19 \\
7.78 \\
10.03 \\
10.06 \\
10.20 \\
10.19\end{array}$ & $\begin{array}{r}96.79 \\
100.53 \\
99.36 \\
98.89 \\
100.14 \\
99.57\end{array}$ & $\begin{array}{r}10.57 \\
8.44 \\
10.66 \\
11.05 \\
9.75 \\
11.13\end{array}$ & $\begin{array}{l}0.700 \\
0.318 \\
0.288 \\
0.664 \\
0.880 \\
0.644\end{array}$ \\
\hline $\begin{array}{r}\text { Maternal Height }(\mathrm{Cmt}) \\
\text { Pre-Pregnancy Weight }(\mathrm{kgr}) \\
\text { Gestational Age } \\
\text { Maternal Social Support }\end{array}$ & $\begin{array}{c}164.30 \\
62.83 \\
16.34\end{array}$ & $\begin{array}{r}7.47 \\
14.13 \\
5.72\end{array}$ & $\begin{array}{r}164.49 \\
61.22 \\
16.31\end{array}$ & $\begin{array}{r}6.46 \\
12.03 \\
5.47\end{array}$ & $\begin{array}{l}0.809 \\
0.273 \\
0.972\end{array}$ & $\begin{array}{r}164.89 \\
61.31 \\
16.75\end{array}$ & $\begin{array}{r}6.98 \\
15.57 \\
5.82\end{array}$ & $\begin{array}{r}163.60 \\
63.39 \\
17.07\end{array}$ & $\begin{array}{r}6.66 \\
14.67 \\
5.83\end{array}$ & $\begin{array}{l}0.109 \\
0.233 \\
0.644\end{array}$ \\
\hline $\begin{array}{l}\text { Grandmother Social Support } \\
\text { Husband/Boyfriend Social Support } \\
\text { Maternal Risky Behaviors }\end{array}$ & $\begin{array}{l}99.04 \\
99.81\end{array}$ & $\begin{array}{l}10.74 \\
10.20\end{array}$ & $\begin{array}{r}101.22 \\
99.96\end{array}$ & $\begin{array}{l}9.60 \\
9.77\end{array}$ & $\begin{array}{r}\mathbf{0 . 0 5 9} \\
0.891\end{array}$ & $\begin{array}{l}100.99 \\
100.42\end{array}$ & $\begin{array}{l}8.30 \\
9.98\end{array}$ & $\begin{array}{l}101.86 \\
101.07\end{array}$ & $\begin{array}{r}7.91 \\
10.25\end{array}$ & $\begin{array}{l}0.355 \\
0.585\end{array}$ \\
\hline $\begin{array}{r}\text { Alcohol Consumption (Past } 2 \mathrm{wks}) \\
\text { Smoking (Past } 3 \text { days) } \\
\text { Used Marijuana (Past } 2 \mathrm{wks}) \\
\text { Sexually Transmitted Diseases }\end{array}$ & $\begin{array}{l}0.033 \\
0.090 \\
0.025 \\
0.335\end{array}$ & $\begin{array}{l}0.178 \\
0.287 \\
0.271 \\
0.473\end{array}$ & $\begin{array}{l}0.064 \\
0.128 \\
0.018 \\
0.349\end{array}$ & $\begin{array}{l}0.246 \\
0.336 \\
0.192 \\
0.479\end{array}$ & $\begin{array}{l}0.232 \\
0.303 \\
0.805 \\
0.801\end{array}$ & $\begin{array}{l}0.046 \\
0.101 \\
0.038 \\
0.324\end{array}$ & $\begin{array}{l}0.210 \\
0.302 \\
0.323 \\
0.469\end{array}$ & $\begin{array}{l}0.028 \\
0.093 \\
0.112 \\
0.402\end{array}$ & $\begin{array}{l}0.166 \\
0.292 \\
1.160 \\
0.493\end{array}$ & $\begin{array}{l}0.389 \\
0.830 \\
0.516 \\
0.167\end{array}$ \\
\hline
\end{tabular}

Notes: This table presents the statistical description of selected pre-program variables at baseline. The first column of the table gives the variable description. The variables are divided into groups that share similar meanings. The remainder of the table consists of the description of the blocks of variables associated with the whole sample, the female sample and the male sample. Each block has 6 columns: (1) Control mean, (2) Control standard deviation, (3) Treatment mean, (4) Treatment standard deviation and (5) Asymptotic $p$-value associated with the difference in means. Bold $p$-values indicate that the t-statistic between the control and the treatment means is significant at the $10 \%$ level. Maternal social support corresponds to standardized indices. Additional baseline tables using samples at years 6 and 12 can be found in Section E of the Appendix. 
12 years old, $86 \%$ of mothers who had no fetal or child death were interviewed. ${ }^{7}$

\section{Baseline Information}

We use race, mother's age, marital status, family income, employment, education, fertility, delinquency, maternal mental health, family support, and maternal risk behavior as background information.

\section{Outcomes}

Data from the NFP Memphis trial were collected at multiple time points including birth (e.g., placenta and birth weight) and when the child was 6 months, 1 year, 2 years, 6 years, and 12 years old. Table 3 summarizes the data available for each period of child development. See Appendix C for a detailed description of each of the NFP measures. Data on home environment (HOME) and parenting skills (Bavolek) were collected at 6 months, 1 year, and 2 years old. ${ }^{8}$ Data on socio-emotional skills (CBCL), hospital records ${ }^{9}$, and maternal mental health are available at age $2 .{ }^{10}$ Maternal mental health was assessed at age 2 by the Rand Mental Health Inventory and the Rosenberg and Pearlin Scales. ${ }^{11}$ Child cognition (KABC, PPVT, WISC-III) ${ }^{12}$ and socio-emotional skills (CBCL, MSSB) were measured at age $6 .{ }^{13}$ Outcomes at age 12 consist of child's internalizing and externalizing behaviors (CBCL),

\footnotetext{
${ }^{7}$ Tables E.1-E.3 in Appendix E present a statistical description of retention levels and baseline variables. Table E.1 presents the statistical description of retention levels by gender and time of survey. Table E.2 presents the statistical description of selected pre-program variables after 6 years. Table E.3 investigates the distribution of baseline variables after 12 years.

${ }^{8} \mathrm{HOME}$ stands for the Home Observation for Measurement of the Environment. It measures the resources and time allocated to cognitive and emotional stimuli. Bavolek is the Adult-Adolescent Parenting Inventory that measures parental skills and child-rearing attitudes such as abusive and neglecting parenting.

${ }^{9}$ Those include health care usage such as hospitalizations and medical appointments.

${ }^{10}$ Child-Behavior Checklist (CBCL) measures child behavioral and emotional problems reported by the mother (Association and on DSM-IV., 2000).

${ }^{11}$ These instruments measure maternal psychological skills. The Rosenberg Self Esteem Scale assesses global self-worth (Rosenberg, 1965). The Pearlin Mastery Scale measures personal mastery over life outcomes (Pearlin and Schooler, 1978). The Rand Mental Health Inventory captures psychological distress and wellbeing factors including anxiety, depression, and positive well-being (Veit and Ware, 1983).

${ }^{12} \mathrm{KABC}$ stands for the Kaufman Assessment Battery for Children and focuses on problem solving and language skills. PPVT stands for Peabody Picture Vocabulary Test (PPVT) and tests receptive vocabulary. WISC-III stands for the Wechsler Intelligence Scale for Children (WISC-III) and measures short-term memory and visual perception.

${ }^{13}$ MSSB stands for the McArthur Story Stem Battery which measures storytelling affection and coherence (Olds et al., 2004). MSSB measures four dimensions of socio-emotional skills: (1) child deregulated aggression; (2) parental warmth and empathy; (3) child emotional integration; and (4) child anxiety.
} 
achievement scores (GPA, TCAP, PIAT),${ }^{14}$ and child mental health. We also examine data on parental participation in welfare. ${ }^{15}$

\section{Table 3: NFP Classification of Outcomes/Mediators}

\begin{tabular}{|c|c|c|c|c|c|c|c|c|}
\hline \multirow[b]{2}{*}{ Instrument } & \multicolumn{2}{|r|}{ Content } & \multicolumn{6}{|c|}{ Child's Age } \\
\hline & Subject & Classification & 0 & 0.5 & 1 & 2 & 6 & 12 \\
\hline Birth Biometrics & | Child/Mother & Health & $\checkmark$ & & & & & \\
\hline Hospital Visits & Child & Health & $\checkmark$ & $\checkmark$ & $\checkmark$ & $\checkmark$ & & \\
\hline Non-Abusive Parenting (Bavolek) & Parents & Environment/Parenting & & $\checkmark$ & $\checkmark$ & $\checkmark$ & & \\
\hline Home Environment Score (HOME) & Family & Environment/Parenting & & & $\checkmark$ & $\checkmark$ & & \\
\hline Maternal Mental Health & Mother & Mental Health & & & & $\checkmark$ & & \\
\hline Kaufman Assessment (KABC) & Child & Cognition & & & & & $\checkmark$ & \\
\hline Peabody Picture Vocabulary Test (PPVT) & Child & Cognition & & & & & $\checkmark$ & \\
\hline Wechsler Intelligence Scale (WISC-III) & Child & Cognition & & & & & $\checkmark$ & \\
\hline Child Behavior Checklist (CBCL) & Child & Socio-emotional & & & & $\checkmark$ & $\checkmark$ & \\
\hline MacArthur Story Stem Battery (MSSB) & Child & Socio-emotional & & & & & $\checkmark$ & \\
\hline Health Injuries & Child & Health & & & & & & $\checkmark$ \\
\hline Body Mass Index (BMI) & Child & Health & & & & & & $\checkmark$ \\
\hline Peabody Individual Achievement Test (PIAT) & Child & Achievement Scores & & & & & & $\checkmark$ \\
\hline Tennessee Comprehensive Assessment (TCAP) & Child & Achievement Scores & & & & & & $\checkmark$ \\
\hline Schooling Grades (GPA) & Child & Achievement Scores & & & & & & $\checkmark$ \\
\hline CBCL/Youth Self-Report & Child & Mental Health & & & & & & $\checkmark$ \\
\hline Welfare Use & Family & Governmental Aid & & & & & & $\checkmark$ \\
\hline
\end{tabular}

Notes: This table presents a summary of the data available for the analysis of treatment effects in NFP mediators and outcomes. The first column presents the name of the outcome or instrument. The remaining columns specify the time of survey according to the age of the child in participating families. Additional information on the description of the available instruments of the NFP data can be seen at Appendix C.

\section{Inference}

Previous literature uses classical inference that relies on large sample (asymptotic) assumptions to make statistical inference about NFP. Heckman et al. (2010) show that the classical approach may generate biased inference when applied to small sample sizes, if the distribution of the data is skewed or, more generally, non-normal. Permutation-based tests provide a solution to these potential problems.

Permutation tests are often called distribution-free as they do not require parametric assumptions about the data-generating process and are valid for any sample size. Permutation

\footnotetext{
${ }^{14}$ GPA records from grades 1 to 5 were obtained through school records. TCAP stands for the Tennessee Comprehensive Assessment Program, an achievement test administered by Tennessee schools after 3rd grade. PIAT stands for Peabody Individual Achievement Test.

${ }^{15}$ Obtained from the Tennessee administrative records.
} 
tests exploit an exchangeability property that arises from the randomization protocol. If the null hypothesis of no treatment effect is true, then permuting treated and control outcomes within strata (blocks) formed by the variables used in the randomization protocol should not alter the distribution of the observed outcomes. This insight enables us to evaluate the $p$-value for the null hypothesis of no treatment effect by using the fraction of the re-sampled data that generates a test statistic greater than the one obtained from the original data. This procedure is called a block-permutation test. In the case of NFP, the baseline variables used in the randomization protocol are: maternal age and race, gestational age at enrollment, employment status of the head of the household, and geographic region. See Appendix D for a detailed discussion of the permutation test we use.

Another concern in evaluating and testing treatment effects is the imbalance of baseline characteristics across treatment and control groups. We address this issue by assuming linearity in background variables in the outcome equations to control for imbalances. We use the approach suggested by Freedman and Lane (1983) which, according to Anderson and Legendre (1999), provides the most reliable results among a range of permutation methods for linear models. See Appendix D.1 for a detailed description of this method. We control for maternal height, household income, grandmother support, maternal parenting attitudes, and whether the mother is currently enrolled in school.

In addition, the large number of outcomes associated with NFP creates the possibility of "cherry picking," namely the selective reporting of statistically significant treatment effects that may occur by chance. We account for the multiplicity of outcomes by using a multiplehypothesis testing procedure based on the stepdown method of Romano and Wolf (2005). The method generates $p$-values that control for the Family-wise Error Rate (FWER): that is, the probability of rejecting at least one true hypothesis out of a set of multiple hypotheses. Stepdown $p$-values are more conservative than single hypothesis $p$-values, since they take into account the multiplicity of outcomes. For a detailed description of the stepdown method, see Appendix D. 
Table 5 presents a summary of the inference analysis and compares the number of statistically significant outcomes using three methods of inference. The first method is classical inference for single-hypotheses, which relies on large sample assumptions. The second method is the single-hypothesis block-permutation test previously discussed. The third method applies the stepdown method, which accounts for multiple outcomes. We find that correcting for the randomization protocol and the imbalance of baseline variables using permutation tests often produces sharper inferences compared to those obtained from classical inference procedures. As expected, fewer outcomes survive corrections for multiple-hypothesis testing. The next section explain the results of our inference reanalysis in more detail.

\section{Estimated Treatment Effects}

Table 4 presents a synopsis of the overall significance of the NFP treatment effects. The table shows the percentage of outcomes for which treatment effects are statistically significant at different significance levels by gender. These percentages are larger than what would be expected to arise by chance.

Table 4: Percentage of Test Statistics Exceeding Various Significance Levels

\begin{tabular}{c|cc}
\hline Significance Level & Female Sample Male Sample \\
\hline & & \\
Percentage of $p$-values smaller than 1\% & $7.6 \%$ & $2.5 \%$ \\
Percentage of $p$-values smaller than 5\% & $22.3 \%$ & $17.2 \%$ \\
Percentage of $p$-values smaller than 10\% & $33.8 \%$ & $35.7 \%$ \\
& & \\
\hline
\end{tabular}

Note: This table presents the percentage of statistically significant treatment effects based on a selection of 157 outcomes in the NFP study. Among those, 5 outcomes are surveyed at birth; 1 at age 6 months old; 2 at age 1; 51 outcomes are surveyed at age $2 ; 37$ outcomes are surveyed at age $6 ; 3$ outcomes are surveyed at age 9 ; and 58 outcomes are surveyed at age 12 .

Table 5 summarizes the inference results on treatment effects presented in Tables 6- 
10. According to Table 5, the NFP intervention significantly improved maternal mental health, home environment and parenting skills. On average, treated boys were healthier at birth and experienced an increase in cognitive abilities by age 6 . We find that the NFP intervention generated stronger effects on socio-emotional skills for girls and stronger effects on academic achievement for boys. By age 12, treated boys outperformed controls in math and reading achievement. Treated girls experienced an improvement in cognitive and socioemotional skills at age 6 . At age 12, treated girls had a lower body-mass index (BMI), but the estimated treatment effects are not significantly different from the control group in other measures. We also find that controlling for background characteristics increases the precision of treatment effect estimates, often leading to statistical significance in multiple-hypothesis inference. ${ }^{16}$ Tables F.7-F.10 of Appendix F show that our findings hold when correcting for attrition using the inverse probability weighting method. ${ }^{17}$ Treatment effect estimates and inferences follow closely the results presented in Tables 6-10. We conclude that the correction for attrition does not play a substantial role in the evaluation of the NFP intervention.

Tables 6-10 display the estimation and inference of treatment effects by outcome category and gender. We divide outcomes into five categories: child health, family environments, child cognition, socio-emotional development, and achievement scores. Outcomes are divided into blocks of variables within each category. Each block contains variables of similar content surveyed at the same period. Each table consists of six columns for each gender. The first four columns display the basic statistics for each block of outcomes: (1) control group mean, (2) conditional difference in mean, (3) conditional effect size, and (4) (classic) asymptotic $p$-value. The fifth column presents the one-sided single hypothesis permutation $p$-value that accounts for the characteristics of the NFP randomization protocol as described in Section 2. The sixth column shows the adjusted stepdown $p$-values that correct for multiple-hypothesis testing. In the rest of this section we discuss each outcome category in more detail.

\section{Child Health Outcomes}

\footnotetext{
${ }^{16}$ Tables F.1-F.5 of Appendix F present the unconditional analysis of the conditional treatment effects. ${ }^{17}$ See, e.g., Robins et al. (1994).
} 


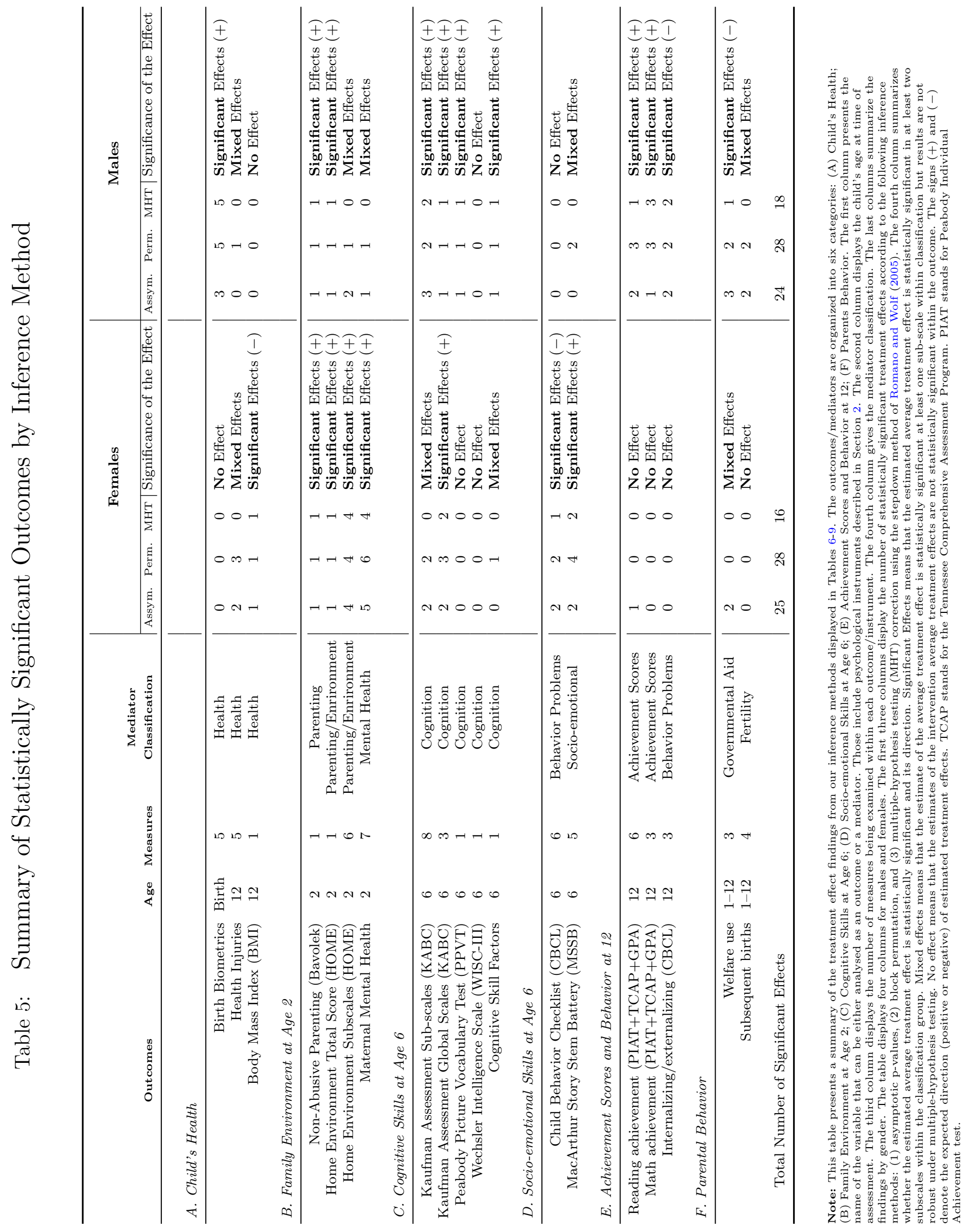


Table 6 displays the NFP results on child health. The first block shows treatment effects on birth outcomes. Treated boys were relatively healthier at birth: there are strong positive effects on placenta weight, birth weight, head circumference, length and gestational age at delivery. The results are both statistically significant at the $5 \%$ significance level and robust to correcting for multiple-hypothesis testing. Treated boys are, on average, 193 grams heavier and were delivered 0.7 weeks later than their control counterparts. In contrast, there are no statistically significant effects for girls at birth. The second block focuses on child health outcomes at age 12. We find statistically significant treatment effects on body mass index (BMI) for girls. Boys have fewer statistically significant results at age 12, which do not survive multiple-hypothesis corrections.

\section{Family environment}

Table 7 displays results on family environments and parenting. The first two blocks display treatment effects on parenting beliefs and the home environment for ages 1 and 2. Treatment effects are statistically significant for both genders and robust to application of the stepdown procedure. By age 2, the NFP improved maternal parenting attitudes regarding non-abusive and non-neglecting behaviors (Bavolek Inventory). We find effect sizes of approximately 0.3 standard deviations (SDs) for mothers of both females and males. In addition, the intervention improved home environments by 0.17 SDs for males and by 0.3 SDs for females. This positive impact on home environments is mainly reflected in treatment effects on HOME sub-scales associated with variety in daily stimulation, and provision of appropriate playing material. Additionally, we find statistically significant treatment effects on maternal mental health. Mothers of female children reported less anxiety, and better emotional stability, self-esteem, and mastery skills. The effects range between 0.2 and 0.3 SDs. Mothers of male children also experienced an improvement in their mastery skills. However, this effect does not survive multiple-hypothesis corrections. The bottom block examines the treatment effects on the cost of welfare program participation. The NFP reduced the total cost of participation in AFDC/TANF, SNAP (food stamps), and Medicaid 
for mothers with male children at age 12. Reduction in SNAP enrollment survive the multiple hypothesis correction for males. Additionally, one of the NFP program objectives was to improve maternal planning of future pregnancies. Table 8 shows the effects of the program on the probability of having subsequent births after the first child at different periods between ages 0 and 12 . The estimated treatment effects are imprecise and do not survive multiplehypothesis testing correction.

\section{Child Cognitive Outcomes}

Table 9 displays the NFP results on cognition and achievement for children at ages 6 and 12. The first block analyzes the Kaufmann Assessment Battery for Children (KABC) inventory measured at age 6 . In particular, it displays the subtests that are part of the mental processing composite (sequential and simultaneous processing). NFP increased IQ for both genders. However, only the results for boys are robust to controlling for multiplehypothesis testing. The second block displays outcomes on some cognitive-related subscales from the $\mathrm{KABC}$, and the results are robust for both boys and girls. Also, treated boys scored 0.25 SDs higher in the PPVT (Peabody Picture Vocabulary Test) than their control counterparts.

To summarize effects on child cognition at age 6 , we estimate two factor scores. The first factor captures both cognition and achievement, which is derived using the three instruments KABC, PPVT and WISC-III. The second factor captures a pure measure of cognitive skills

that comes from the KABC mental processing composite. The treatment effect sizes are larger for boys than girls. For instance, NFP increased cognitive skills by 0.27 SDs for boys versus 0.12 SDs for girls.

\section{Achievement Scores}

The bottom part of Table 9 presents the treatment effects on achievement outcomes at age 12 (reading and math). The results, again, are stronger for treated boys than treated 
girls, and are robust to the application of the stepdown procedure. Treated boys scored 0.24 SDs higher in the language section of an achievement test: the Tennessee Comprehensive Assessment Program (TCAP). Also, NFP improved math achievement scores exclusively for boys. Treatment effect sizes range between 0.15 and 0.22 SDs. In general, Table 9 documents a wide variety of effects on the cognitive skills of boys and girls. These results extend previous evaluations, which found this effect only for children born to mothers with low psychological resources (Kitzman et al., 2010; Olds, 2012)

\section{Child Socio-Emotional Outcomes}

Table 10 displays the NFP results on socio-emotional development. The upper blocks investigate the treatment effects on psychosocial functioning based on the Child Behavior Checklist (CBCL) at ages 2 and 6 . The results are statistically significant only for treated girls, who had fewer affective and attention problems at age 2 (effect size: $0.24-0.33$ SDs) and fewer conduct and attention problems at age 6 (effect size: 0.27 SDs). Also, children's socioemotional skills were assessed using the MacArthur Story Stem Battery (MSSB) surveyed at age 6 . We find that treated girls improved pro-social skills (warmth and empathy) by 0.36 SDs and decreased aggressive behavior by 0.18 SDs. The bottom block of Table 10 considers socio-emotional outcomes at age 12. Treated boys experienced less internalizing disorders and fewer school absences (effect sizes of approximately 0.2 SDs). NFP treatment effects differ by gender. Socio-emotional effects are stronger for girls while treatment effects on academic achievement are statistically significant for boys.

Our results are consistent with the existing literature on home visiting programs that have shown positive effects on children's socio-emotional development. For example, using reports from teachers at age 5 from the Preparing for Life (PFL) program, Doyle (2017) found reductions in problematic behaviors but not improvement in pro-social skills. The effects sizes ranged between 0.31 and 0.5 SDs. For the case of the NFP, results on socioemotional development at age 6 are found mainly for girls, who experienced a reduction in 
conduct problems and aggression and an improvement in pro-social skills with effect sizes between 0.18 and 0.36 SDs. 


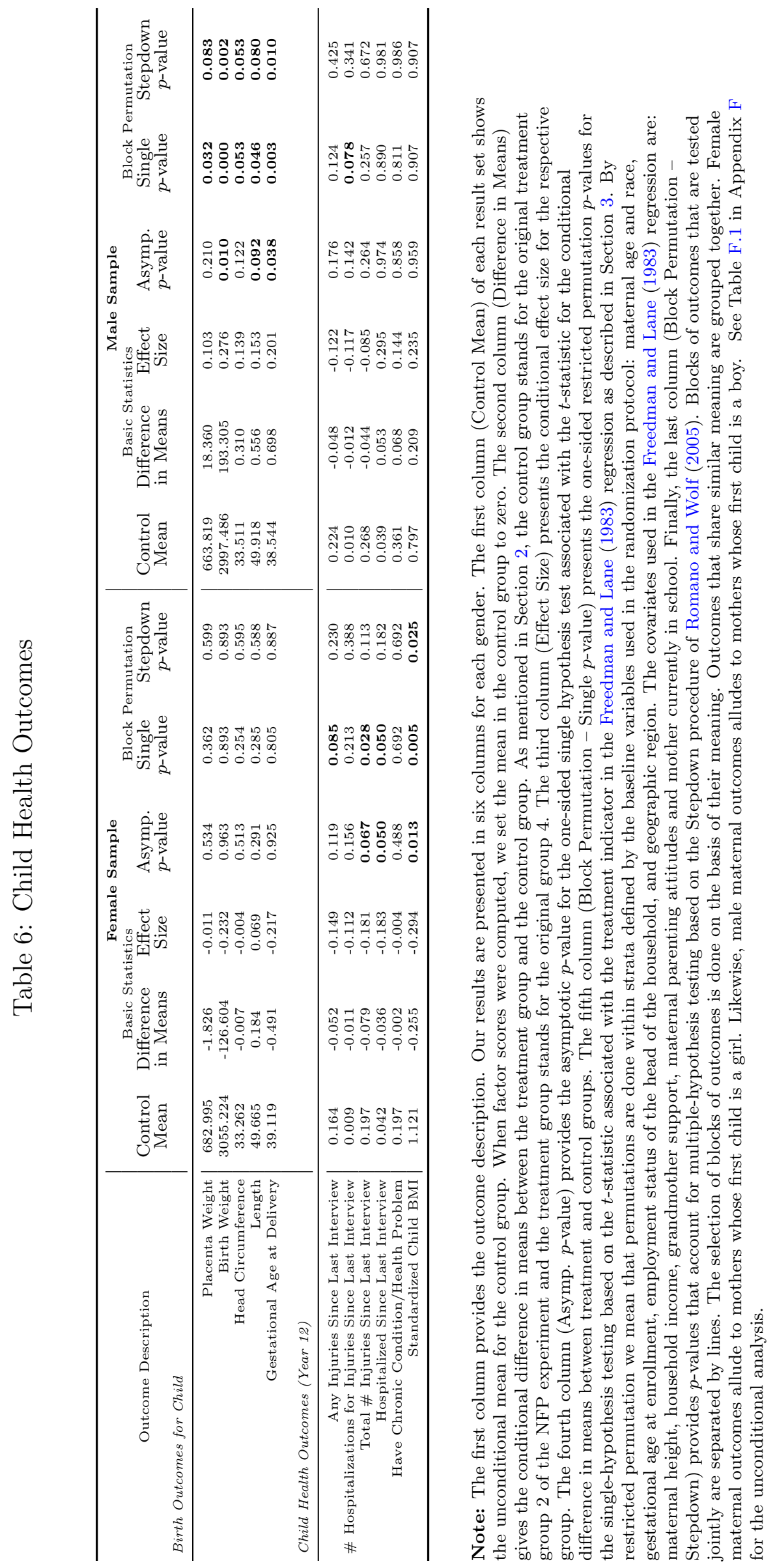




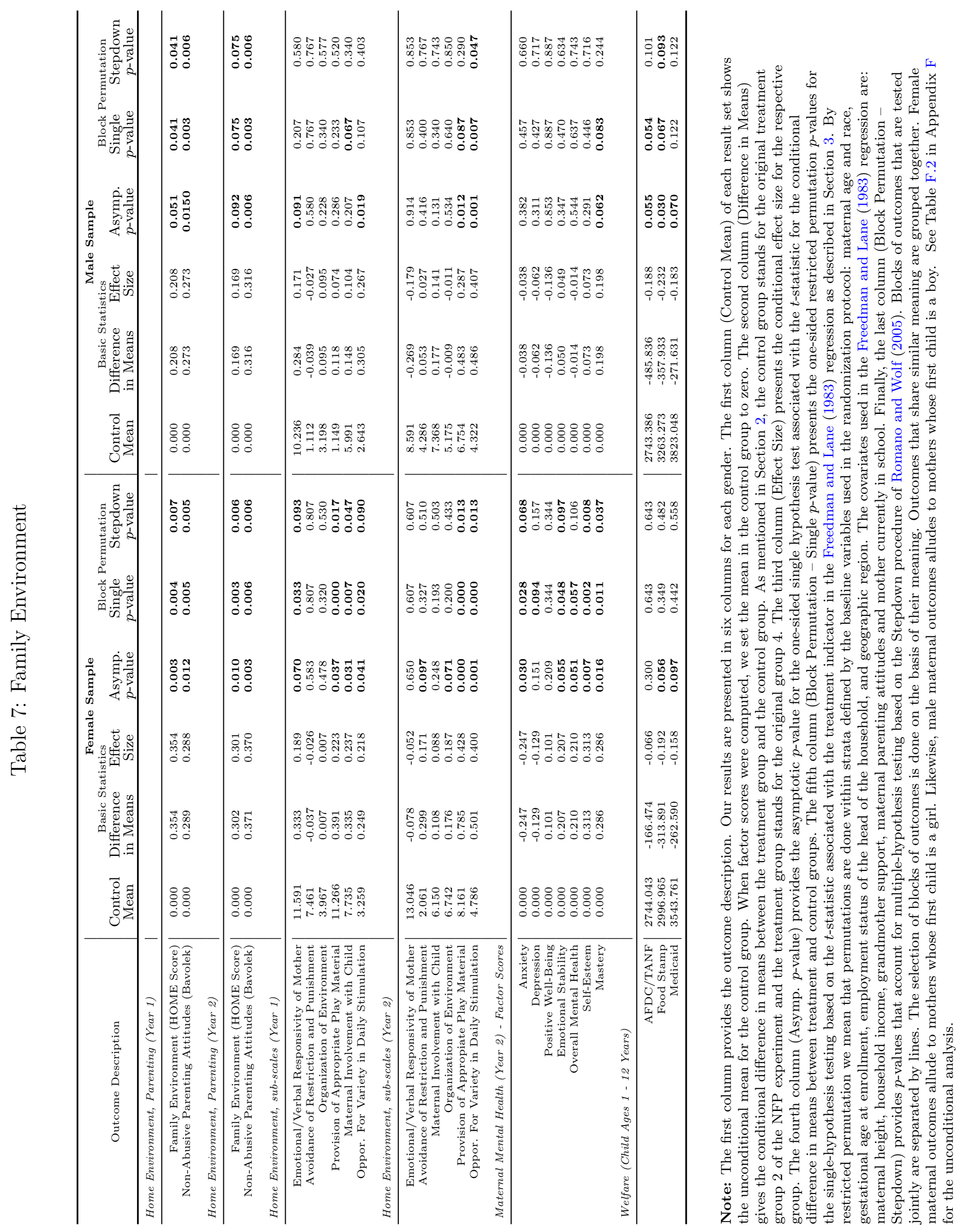




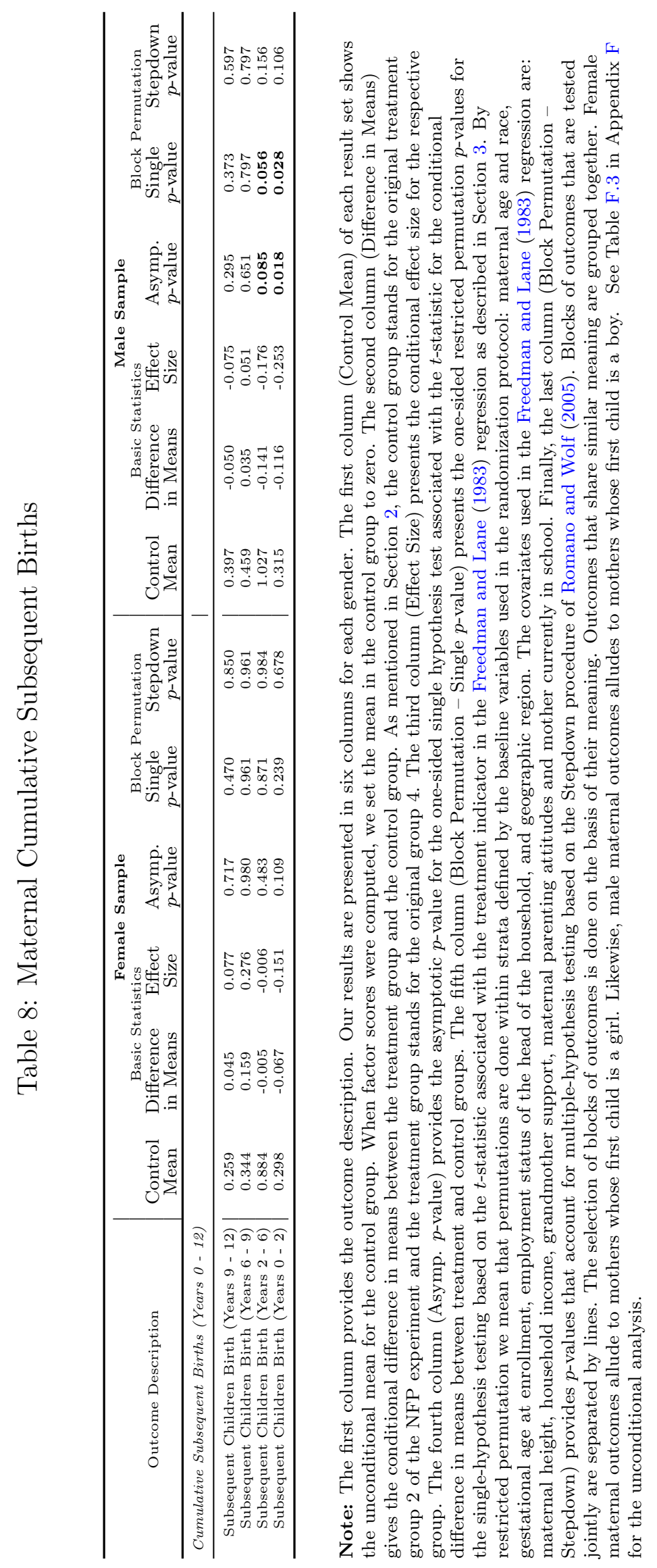




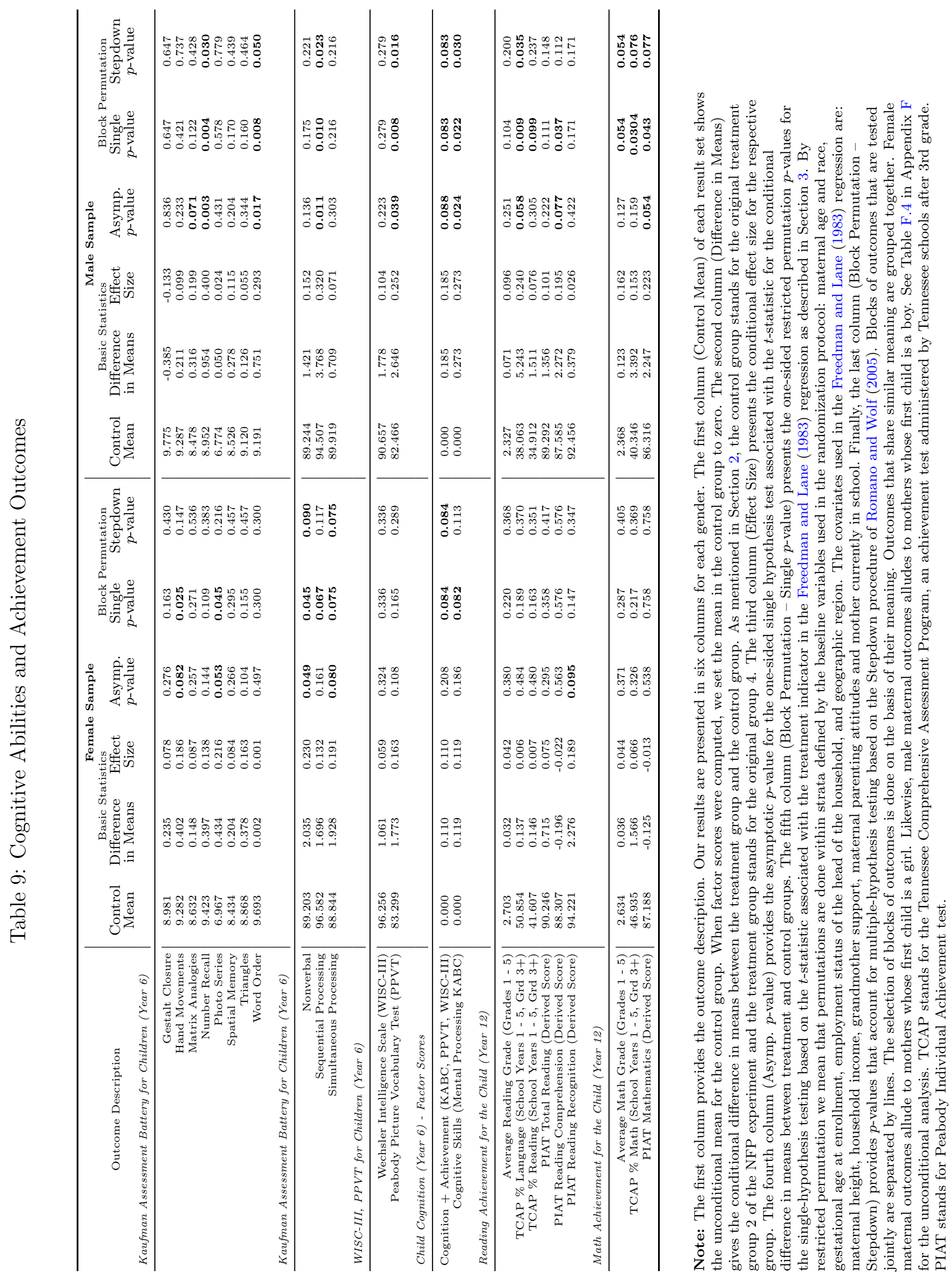




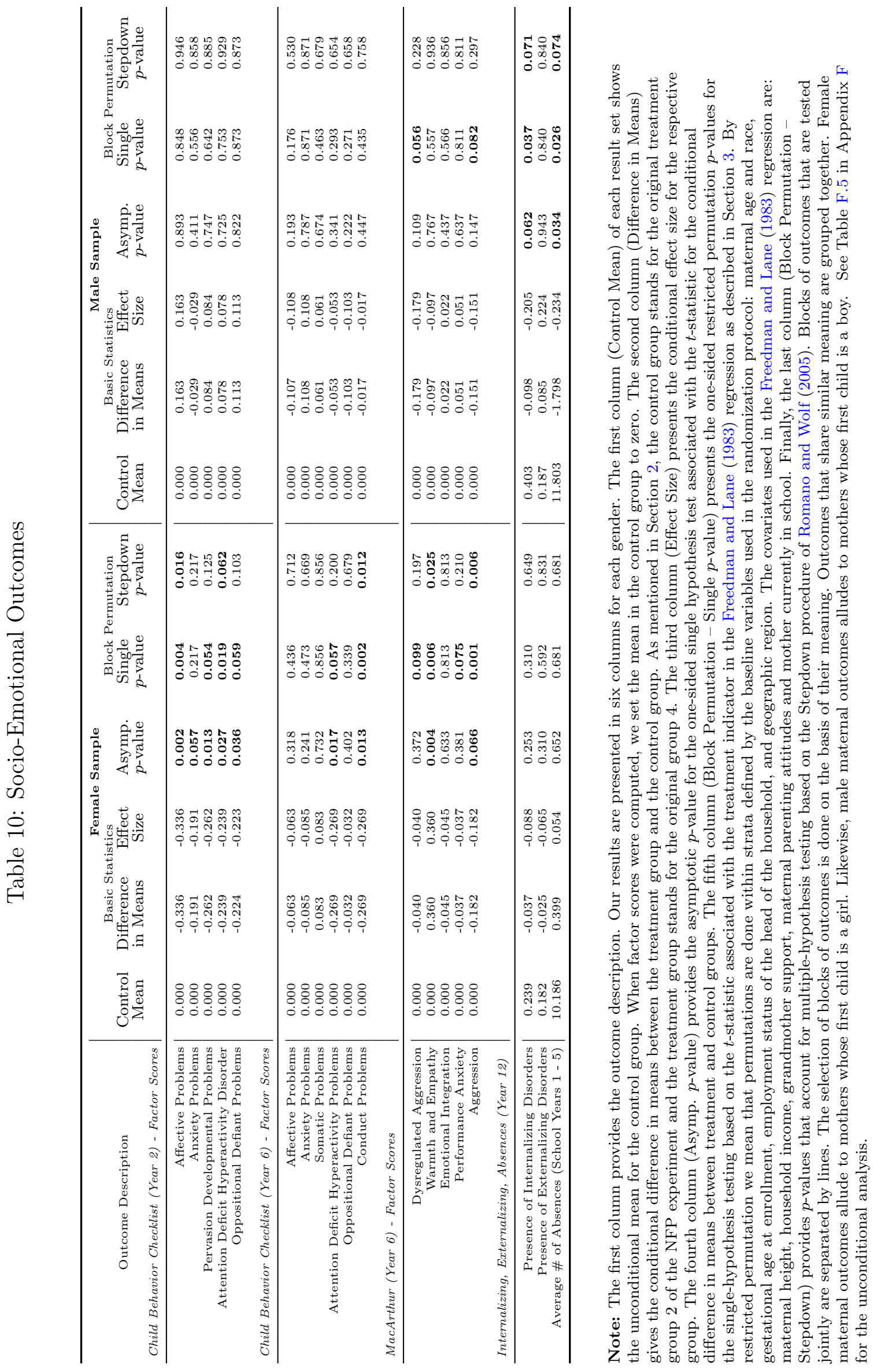




\section{Understanding the Sources of NFP Treatment Ef- fects}

We decompose the NFP treatment effects on pre-pubescent outcomes into interpretable components associated with intervention-induced changes in early-age skills. Such decompositions are sometimes called mediation analyses in the statistics literature. ${ }^{18}$ We follow the approach of Heckman et al. (2013) who adopt a linear approximation of the outcome equation: ${ }^{19}$

$$
Y_{i, d}=\kappa+\underbrace{\boldsymbol{\alpha}_{d}^{m} \boldsymbol{\theta}_{i, d}^{m}}_{\text {measured skills }}+\underbrace{\boldsymbol{\beta}_{d} \boldsymbol{X}_{i}}_{\text {pre-program var. }}+\underbrace{\boldsymbol{\alpha}_{d}^{u} \boldsymbol{\theta}_{i, d}^{u}}_{\text {unmeasured skills }}+\underbrace{\epsilon_{i}}_{\text {error term }}, d \in\{0,1\},
$$

where $Y_{i, d}$ stands for the counterfactual outcome of agent $i$ when the treatment is set to $d \in\{0,1\}: d=1$ for treated and $d=0$ for control. Variables $\left(\boldsymbol{\theta}_{i, d}^{m}, \boldsymbol{\theta}_{i, d}^{u}\right)$ represent the skills that can affect counterfactual outcome $Y_{i, d}$ (e.g., achievement test scores) of measured and unmeasured components (measured skills are those that can be proxied by psychological instruments collected by NFP surveys). $\boldsymbol{X}_{i}$ stands for observed pre-intervention variables that we control for. Parameters $\boldsymbol{\alpha}_{d}^{u}, \boldsymbol{\alpha}_{d}^{m}, \boldsymbol{\beta}_{d}$ denote vectors of coefficients that map changes in skills and pre-program variables into changes in counterfactual outcomes. Variable $\epsilon_{i}$ is an unobserved zero-mean error term that is assumed to be statistically independent of all covariates.

It is useful to rewrite Equation (1) as:

$$
Y_{i, d}=\tau_{d}+\boldsymbol{\alpha}_{d}^{m} \boldsymbol{\theta}_{i, d}^{m}+\boldsymbol{\beta}_{d} \boldsymbol{X}_{i}+\tilde{\epsilon}_{i, d} ; d \in\{0,1\}
$$

where $\tau_{d}=\kappa+\boldsymbol{\alpha}_{d}^{u} \boldsymbol{E}\left(\boldsymbol{\theta}_{i, d}^{u}\right), d \in\{0,1\}$, and $\tilde{\epsilon}_{i, d}=\epsilon_{i}+\boldsymbol{\alpha}_{d}^{u}\left(\boldsymbol{\theta}_{i, d}^{u}-\boldsymbol{E}\left(\boldsymbol{\theta}_{i, d}^{u}\right)\right)$.

Treatment effects arise as a consequence of changes in measured skills $\boldsymbol{\theta}_{i, 1}^{m}, \boldsymbol{\theta}_{i, 0}^{m}$ or changes in

\footnotetext{
${ }^{18}$ See VanderWeele (2015).

${ }^{19}$ See Appendix G for a detailed description of this model.
} 
the mapping between these skills and the outcomes of interest, that is, changes in $\boldsymbol{\alpha}_{1}^{m}, \boldsymbol{\alpha}_{0}^{m}$ or $\tau_{1}, \tau_{0}$. We test whether the map between measured skills and outcomes is the same for treated and control children in Tables I.3 and I.4 of In Appendix I. We do not reject the hypotheses associated with these restrictions. ${ }^{20}$ Thus, we can simplify Equation (2) to:

$$
Y_{i, d}=\tau_{d}+\boldsymbol{\alpha} \boldsymbol{\theta}_{i, d}^{m}+\boldsymbol{\beta} \boldsymbol{X}_{i}+\tilde{\epsilon}_{i, d} l ; d \in\{0,1\}
$$

Let $D_{i} \in\{0,1\}$ to denote the treatment indicator. The observed outcome $Y_{i}$ and observed skills $\boldsymbol{\theta}_{i}^{m}$ for participant $i$ are given by $Y_{i}=D_{i} \cdot Y_{i, 1}+\left(1-D_{i}\right) \cdot Y_{i, 0}$, and $\boldsymbol{\theta}_{i}^{m}=\boldsymbol{\theta}_{i, 1}^{m} D_{i}+$ $\boldsymbol{\theta}_{i, 0}^{m}\left(1-D_{i}\right)$, respectively. In this notation, Equation (3) can be expressed as a linear regression equation:

$$
Y_{i}=\tau_{0}+\tau D_{i}+\boldsymbol{\alpha} \boldsymbol{\theta}_{i}^{m}+\boldsymbol{\beta} \boldsymbol{X}_{i}+\tilde{\epsilon}_{i}
$$

where $\tilde{\epsilon}_{i}=\tilde{\epsilon}_{i, 1} D_{i}+\tilde{\epsilon}_{i, 0}\left(1-D_{i}\right)$, and $\tau=\tau_{1}-\tau_{0}$. Parameter $\tau=\tau_{1}-\tau_{0}$ captures the effect of NFP on outcome $Y$ that is due to the average change of unmeasured skills. This is the mean unexplained or residual effect. The coefficients $\boldsymbol{\alpha}$ map how changes in measured skills $\boldsymbol{\theta}_{i}^{m}$ affect the observed outcome $Y_{i}$. The ordinary least squares estimates of $\boldsymbol{\alpha}$ are unbiased if measured skills $\boldsymbol{\theta}_{i}^{m}$ and the error term $\tilde{\epsilon}_{i}$ are uncorrelated. A sufficient condition for unbiasedness is that measured and unmeasured skills are statistically independent conditioned on $\boldsymbol{X}, D .^{21}$

Appendix I presents robustness tests of the independence relation. We perform these tests using measures available in the data, in the same fashion as Heckman et al. (2013). We decompose the outcome conditional-mean treatment effects of the NFP program into

\footnotetext{
${ }^{20}$ We follow the inference method described in Heckman et al. (2013).

${ }^{21}$ The linearity assumption enables us to require a weaker condition: mean independence.
} 
changes associated with measured and unmeasured skills by

$$
\underbrace{\mathrm{E}(Y \mid D=1, \boldsymbol{X})-\mathrm{E}(Y \mid D=0, \boldsymbol{X})}_{\text {conditional treatment effect }}=\underbrace{\left(\tau_{1}-\tau_{0}\right)}_{\text {unmeasured skills }}+\underbrace{\boldsymbol{\alpha} E\left(\boldsymbol{\theta}_{1}^{m}-\boldsymbol{\theta}_{0}^{m}\right)}_{\text {measured skills }} .
$$

Equation (6) expresses treatment effects as a consequence of skill enhancements that are affected by the program.

Candidates for mediators are skills that are statistically significantly influenced by the program $\left(E\left(\boldsymbol{\theta}_{1}^{m}-\boldsymbol{\theta}_{0}^{m}\right) \neq 0\right)$ and that are related with the outcomes $(\boldsymbol{\alpha} \neq 0)$. The next subsection describes our estimation methodology for decomposing NFP treatment effects.

\subsection{Empirical Strategy to Decompose Treatment Effects}

We estimate a factor model in which measured skills are expressed as factors $\boldsymbol{\theta}_{i}^{m}$ using the range of psychological instruments available in the NFP data. We extract factors to identify the skills that are used to explain final outcomes. Factor models are particularly useful for summarizing large sets of response variables (i.e., psychological instruments) by a small number of latent variables (i.e., skills). Unobserved skills are estimated through a weighted average of relevant observed measures. Factor models outperform other arbitrary indexes by reducing the measurement error associated with factor estimation (Gorsuch, 1983).

We use a three-step procedure employed by Heckman et al. (2013). The first step consists of estimating a linear measurement system in which latent skills are a function of measures, that is, observed item-level data on psychological instruments. In the second step, we use the parameters estimated in the previous step to forecast skills. These forecasts are factor scores obtained by the Bartlett (1937) method. In the third step, we explain later outcomes in terms of earlier skills. Specifically, we use the computed factor scores as covariates in the linear regression represented by Equation (4). See Appendix H for a detailed explanation of the three-step procedure used to secure estimates.

A benefit of our application of the three-step procedure is that it clearly distinguishes 
the effect of the intervention on skills and the effect of the experimentally induced changes in these skills on later outcomes. Our standard errors and $p$-values are computed using bootstrapping, as explained in Appendix H.

The NFP psychological instruments target well-specified personality traits (see Appendix C for a description). We incorporate this fact into our methodology by adopting a measurement system that is based on dedicated measures. By this we mean that each observed measure is linked to a single factor. ${ }^{22}$ We allow the latent factors to be correlated. This approach is termed confirmatory factor analysis (Gorsuch, 1983). Similar to Heckman et al. (2013), we correct for the measurement error that arises from using an estimated factor, i.e., factor scores, instead of the true factor.

We conduct two sets of analyses. We first examine if child skill improvements at age 6 are mediated by variables measured at age 2 . Next, we examine if child outcomes at age 12 are mediated by skills measured at age 6 . The choice of potential mediators corresponds to those intermediate skills where significant treatment effects are found in our inferential analysis. $^{23}$ At age 2, we investigate five classes of mediators: (1) non-abusive parenting attitudes measured by the Bavolek Inventory; (2) home investments measured by the HOME inventory; (3) maternal anxiety from the Rand Mental Health Inventory ${ }^{24}$; (4) maternal self-esteem measured by the Rosenberg Scale; and (5) maternal mastery measured by the Pearlin Scale. At age 6, we examine three broad psychological instruments as mediators: (1) child's cognitive skills measured by the KABC mental processing composite; (2) child's socioemotional skills measured by the Child Behavior Checklist scales of attention and conduct problems; (3) child's warmth, empathy and aggression measured by the MacArthur Story Stem Battery.

\footnotetext{
${ }^{22}$ Heckman et al. (2013) show that the existence of at least three measures for each latent skill guarantees identification.

${ }^{23}$ We perform the analysis in this way since we do not have measures of both skills and investments over time. We only observe parental investments/skills at age 2 and child's skills at age 6 and 12. For instance, we observe parental investments at age 2 (HOME score) but no proxy of parental investments at age 6 . We have child's cognitive skills at age 6 (K-ABC), but not at ages 2 or 12 .

${ }^{24}$ The Rand Mental Health Inventory captures psychological distress and well-being factors. Its subscales include anxiety, depression, and positive well-being (Veit and Ware, 1983).
} 


\section{Decomposing Treatment Effects}

We consider two channels generating NFP treatment effects. The first channel considers maternal investments and home environments as mediators. In this mediation study, we address the question of how parental investments respond to interventions and how such investments affect child skills. The second channel considers how program-induced enhancements of early skills translate to improvements in later skills.

Section 6.1 describes the mediators used in our analysis. Section 6.2 examines whether program improvements in child skills at age 6 are mediated by the effects on health at birth and home investments at age 2. In Section 6.3, we assess if the enhancements of child outcomes measured at age 12 are mediated by program effects on cognitive and socioemotional skills at age 6 .

\subsection{NFP Effects on Skills at Ages 2 and 6}

Figures 1-2 present the kernel densities of the factor scores used as mediators in our analysis. We also display the $p$-value for the single hypothesis inference of no mean treatment effects as described in Section 3.

Figure 1 shows the density of mediators at birth and age 2. It shows that NFP participation significantly increased birth weight of treated boys. At age 2, the NFP intervention significantly improved the quality of home environment for both boys and girls ( $p$-values: 0.07 and 0.00 , respectively). It also significantly reduced abusive/neglecting maternal attitudes ( $p$-values: 0.01 for females and 0.00 for males). The maternal characteristics for mothers of females also improved: maternal anxiety decreased ( $p$-value: 0.03$)$ and maternal self-esteem increased ( $p$-value: 0.00). Maternal mastery skills for mothers of both boys and girls were positively influenced as well.

Figure 2 shows the density of skills at age 6 . For boys, the NFP intervention enhanced cognition and reduced aggression ( $p$-values: 0.02 and 0.08 , respectively). For girls, the 
program reduced attention deficits, conduct problems, and aggression ( $p$-values: 0.06, 0.00, and 0.00 , respectively). NFP also increased cognitive and warmth or empathy skills (prosocial skills) for girls ( $p$-values: 0.08 and 0.01, respectively).

\subsection{Decomposition of Treatment Effects at Age 6}

A large body of evidence corroborates the importance of early parental investments in promoting cognitive and socio-emotional development later in life (Almond and Currie, 2010; Cunha and Heckman, 2007; Heckman, 2008). We present evidence on these claims by examining how early inputs impact skills measured at age 6. These skills are: cognition, warmth/empathy, attention problems, conduct problems, and aggression. We only decompose skills whose treatment effects are statistically significant using the methods explained in Section 5. Therefore, the variables used for conducting the decompositions differ by gender. Figures 3 and 4 decompose the treatment effects on these variables into components associated with changes on factors evaluated at birth and age $2 .^{25}$

We find that cognition at age 6 is enhanced through home environment and parenting practices for boys and girls. Specifically, the program-improved home investments at age 2 explain $35 \%$ of the treatment effect on cognition at age 6 for girls ( $p$-value 0.03$)$. The corresponding number for boys is $22 \%$ ( $p$-value 0.05). Similarly, the enhanced parenting practices explain $14 \%$ of the treatment effect on cognition for girls ( $p$-value 0.08 ). For boys, parenting practices explain 11\% ( $p$-value 0.05). Other contributions to cognition are birth weight and maternal anxiety. ${ }^{26}$ Birth weight gains explain $14 \%$ of the cognitive treatment effect for boys ( $p$-value: 0.06). For girls, maternal anxiety explains $25 \%$ ( $p$-value: 0.08$)$.

We find that the intervention-induced changes in home environments account for $21 \%$ of the treatment effect on female warmth/empathy ( $p$-value: 0.01). ${ }^{27}$ Improvements in home environments also account for $16 \%$ of the reduction in female aggression problems ( $p$-value:

\footnotetext{
${ }^{25}$ The $p$-values are computed using the bootstrap method.

26 Birth weight, a measure that summarizes prenatal investments and fetal development, is usually associated with childhood development (Breslau et al., 1994; Currie and Moretti, 2005).

${ }^{27}$ This controls for birth weight, gender and a range of pre-program baseline characteristics.
} 
0.09). Program enhancement of parenting practices at age 2 explains $9 \%$ of the reduction in attention problems ( $p$-value: 0.05 ) and $11 \%$ of the improvement in female warmth/empathy ( $p$-value: 0.02$)$. For boys, parenting practices explain $8 \%$ of the reduction in aggression (p-value: 0.09).

Maternal skills also influence child development. For girls, the decrease in maternal anxiety explains $14 \%$ of the reduction in conduct problems ( $p$-value: 0.06 ). The contribution of maternal self-esteem is negative and accounts for $29 \%$ of the treatment effect on female warmth and empathy. This result is consistent with research that argues that improvements in self-esteem may increase selfishness (Burr and Christensen, 1992). Conversely, the mother's maternal mastery explains $29 \%$ of the improvement in female warmth/empathy (p-value: 0.09$)$.

\subsection{Decomposition of Treatment Effects at Age 12}

Figures 5-7 decompose statistically significant treatment effects at age 12 into treatmentinduced changes of skills at age 6 . We find that changes in male cognitive abilities at age 6 play a substantial role in explaining achievement scores at age 12. The program's effects on cognition for boys at age 6 explain $41 \%$ of the treatment effects on the Tennessee Comprehensive Assessment Program (TCAP) test for language and 66\% for math ( $p$-value: 0.07). ${ }^{28}$ The improvement of cognitive skills also explains $46 \%$ of the treatment effect on PIAT reading comprehension ( $p$-value 0.04 ) and $51 \%$ of the treatment effect on PIAT math scores ( $p$-value 0.04) for boys. Remarkably, boosts in cognition explain $68 \%$ of the treatment effect in the average math GPA for boys ( $p$-value: 0.08$)$.

Male cognitive gains also explain treatment effects on class absenteeism and internalizing problems. Improvements in cognition account for $24 \%$ of the of the reduction in the average number of day absences between the first and the fifth years of schooling ( $p$-value 0.06). Cognitive gains also explain $17 \%$ of the male treatment effect on internalizing behavior $(p$ -

\footnotetext{
${ }^{28}$ This achievement test was administered at grades 3 and above. In the control group, the mean percentile test score for reading was 38th percentile, and for math 40.3th percentile.
} 
value: 0.06$)$ and $14 \%$ of the results on male anxiety/depression at age 12 ( $p$-value: 0.03$)$. The intervention also reduced aggression at age 6 , which accounts for $12 \%$ of the reduction in the likelihood of being anxious/depressed ( $p$-value: 0.09 see Figure 6). ${ }^{29}$.

The program decreased the likelihood of being overweight for females. The reduction in conduct problems fostered by the program at age 6 explains $19 \%$ of the treatment effect on standardized female BMI ( $p$-value: 0.06). When we decompose the treatment effects on female risky behavior at age 12 , we find that this treatment effect is largely unexplained by our mediators (not displayed in the figures). ${ }^{30}$

The differential treatment effects on cognition and achievement by gender follow the pattern of treatment effects found on health at birth, which are stronger for boys. Epidemiological and medical literature has argued that boys are more susceptible to prenatal influences than girls (Kraemer, 2000; Schore, 2017), which could explain why we find effects of the program on birth weight only for boys.

\footnotetext{
${ }^{29}$ Other related results are shown in Tables H.1-H.4 in the Appendix H

${ }^{30}$ They are shown in Table H.3 in the Appendix H
} 


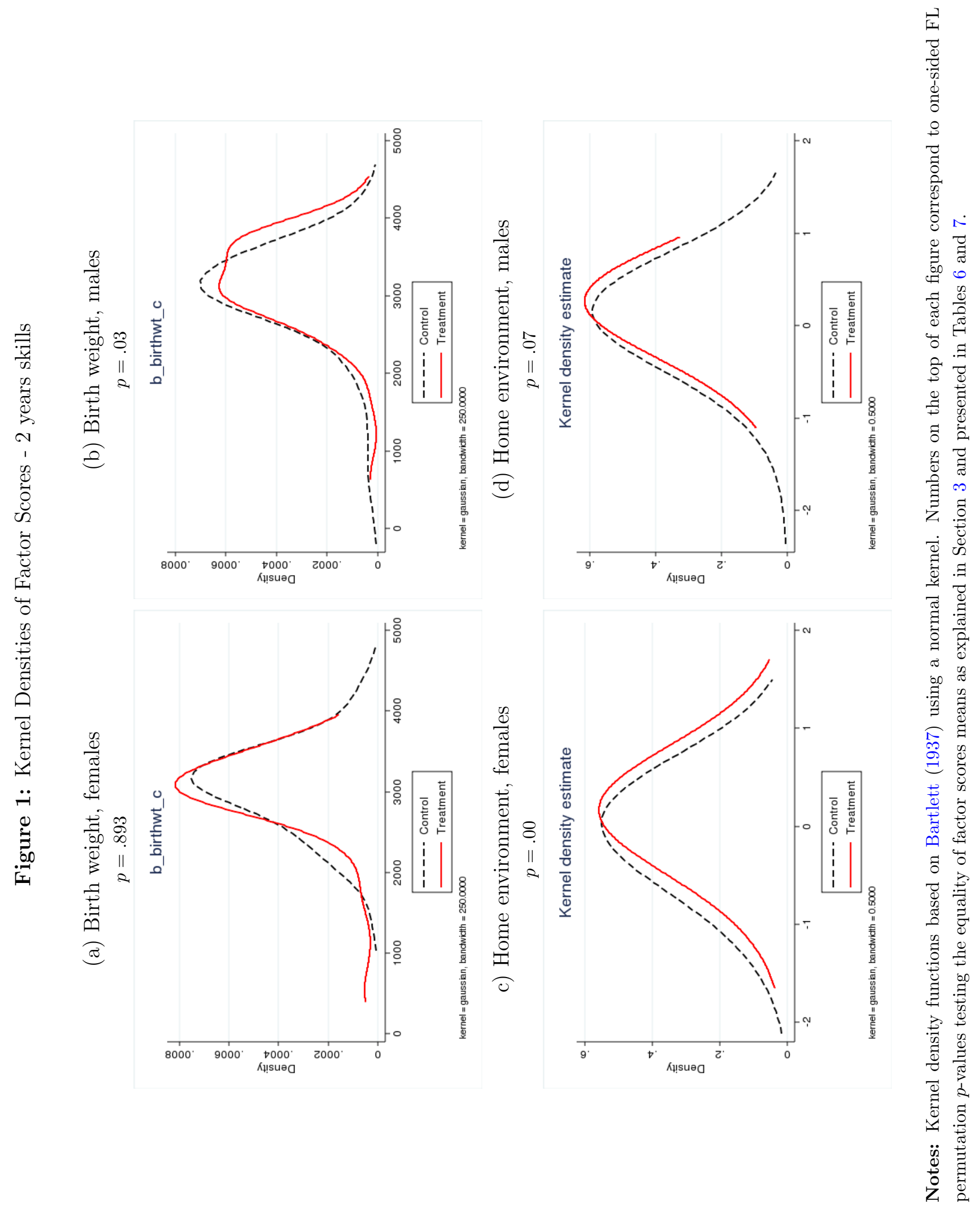




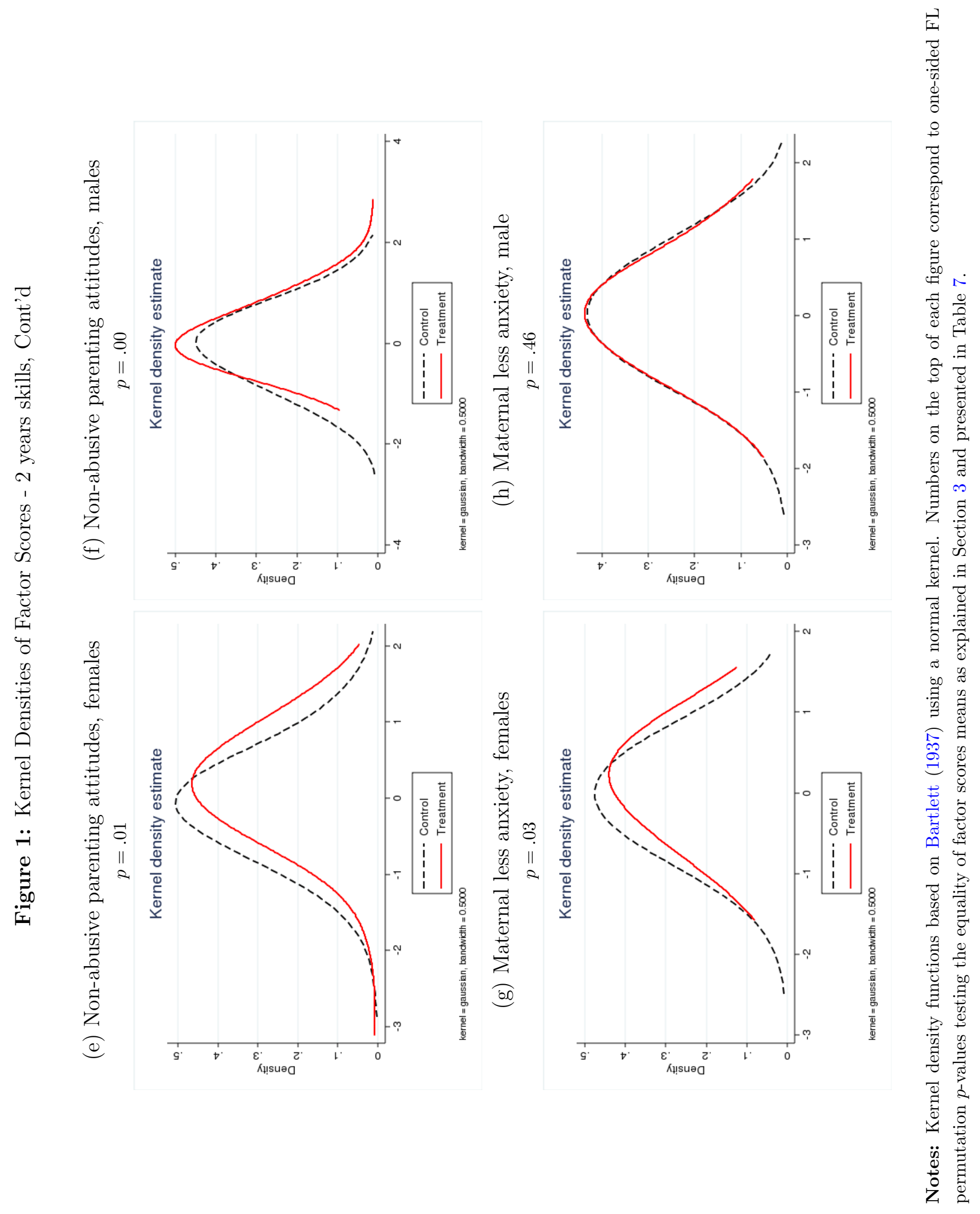




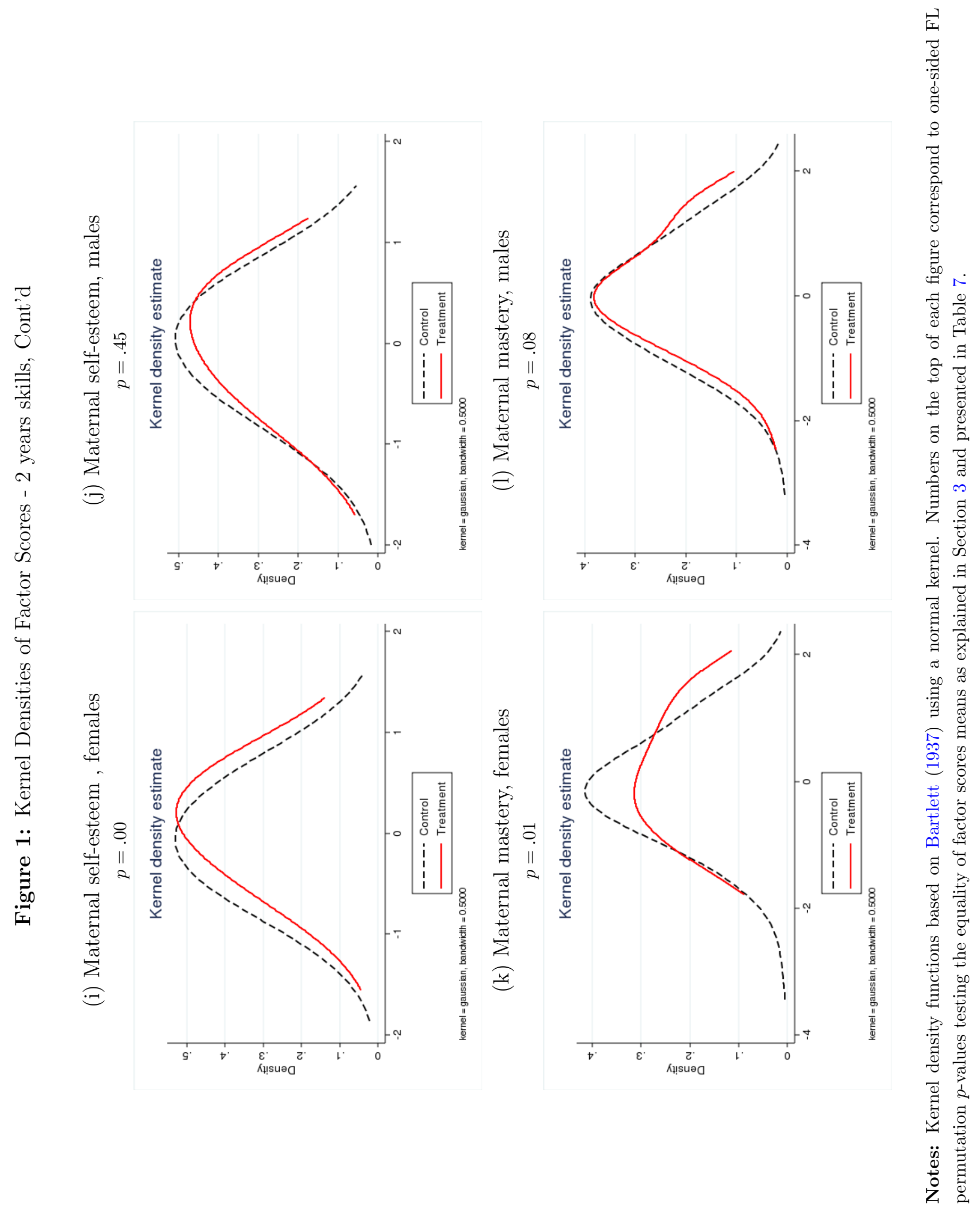




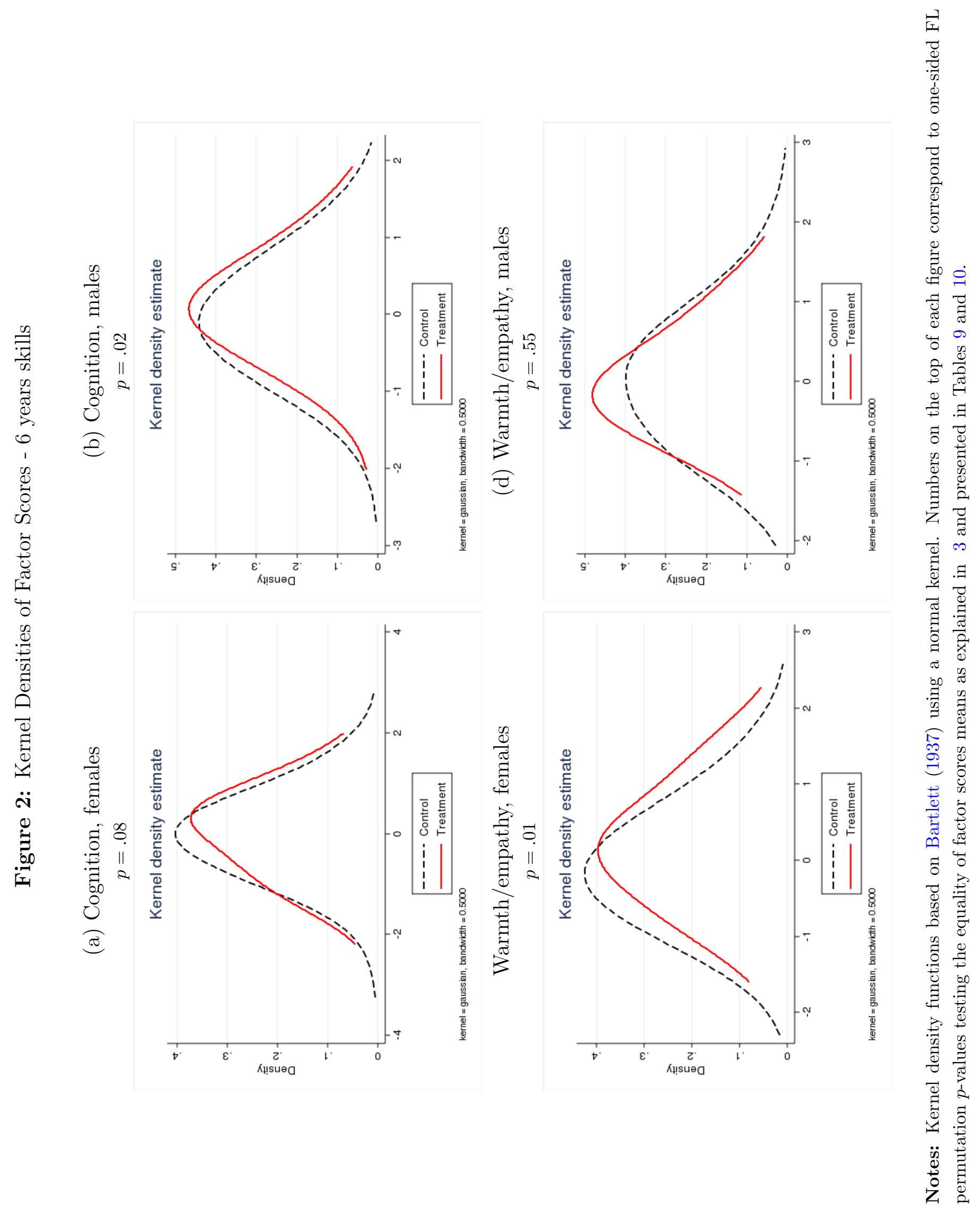




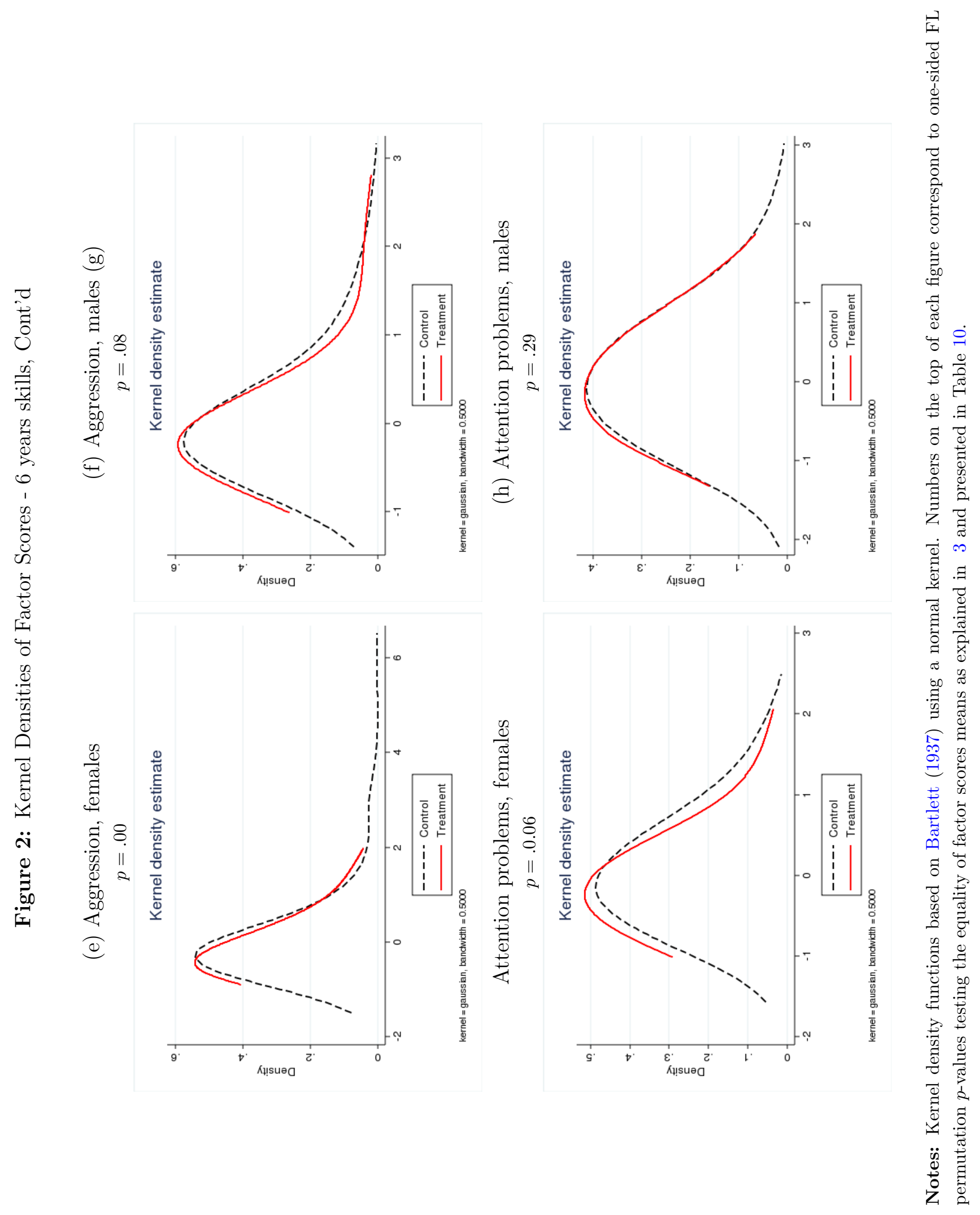




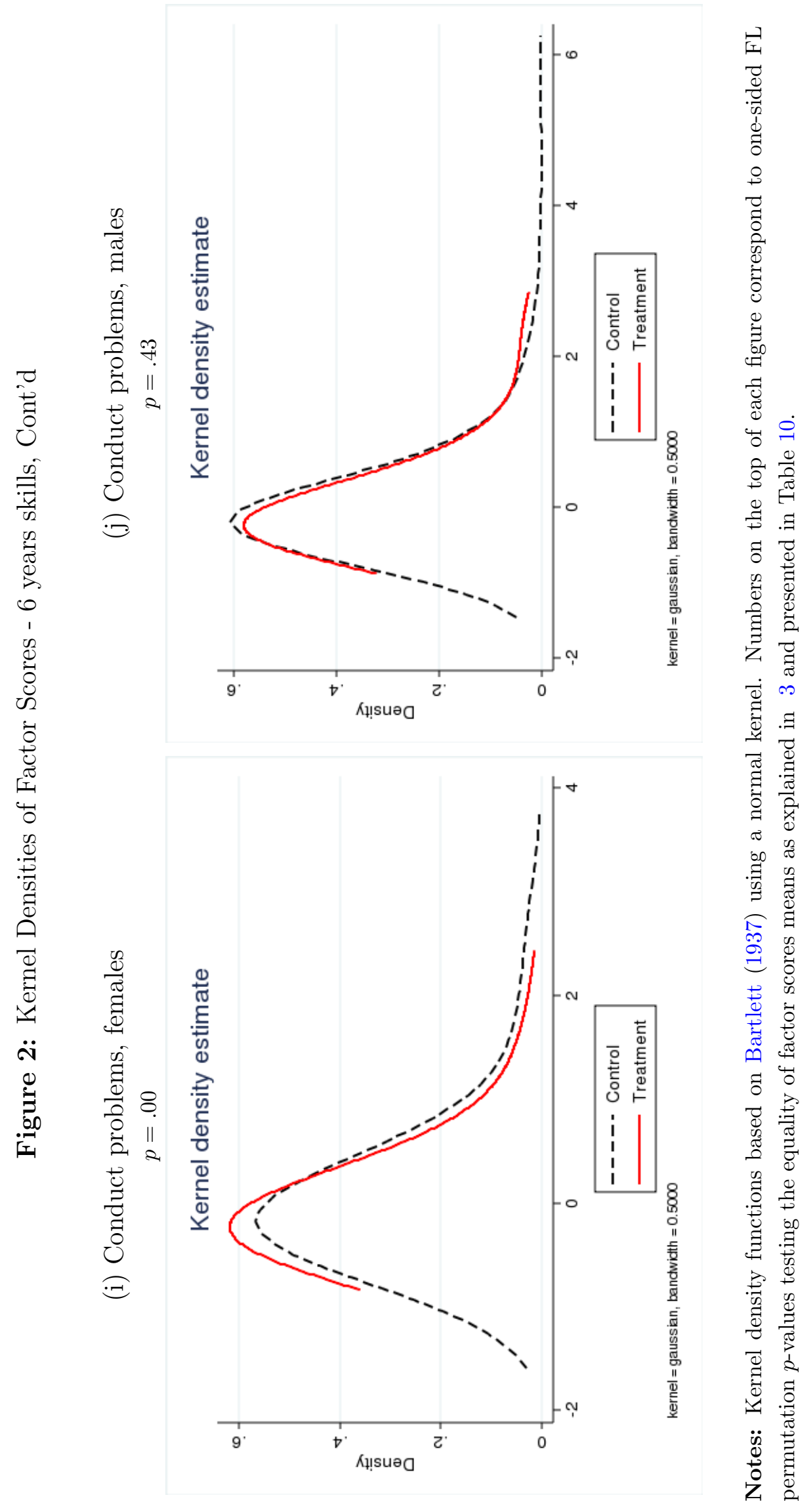




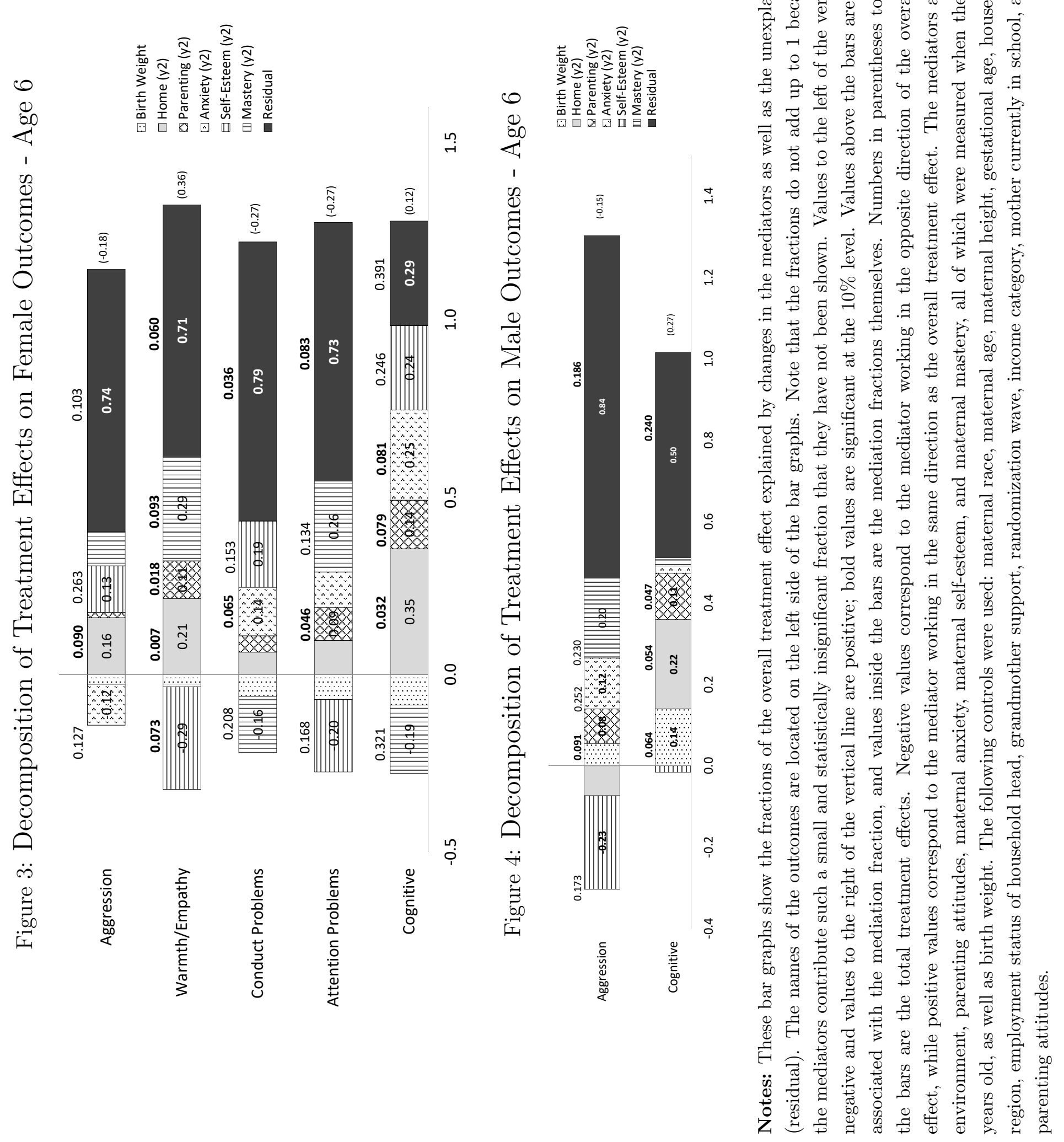




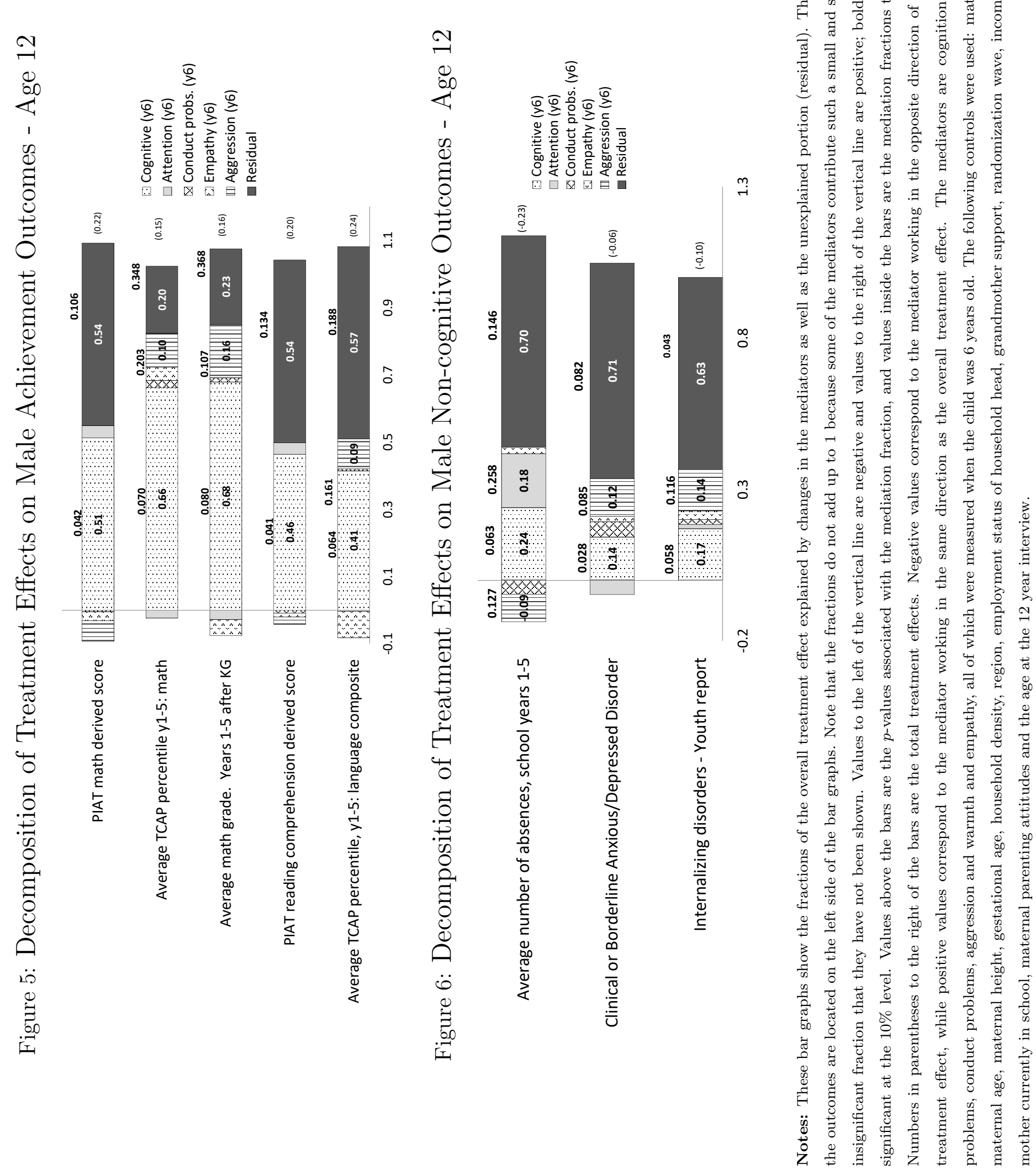




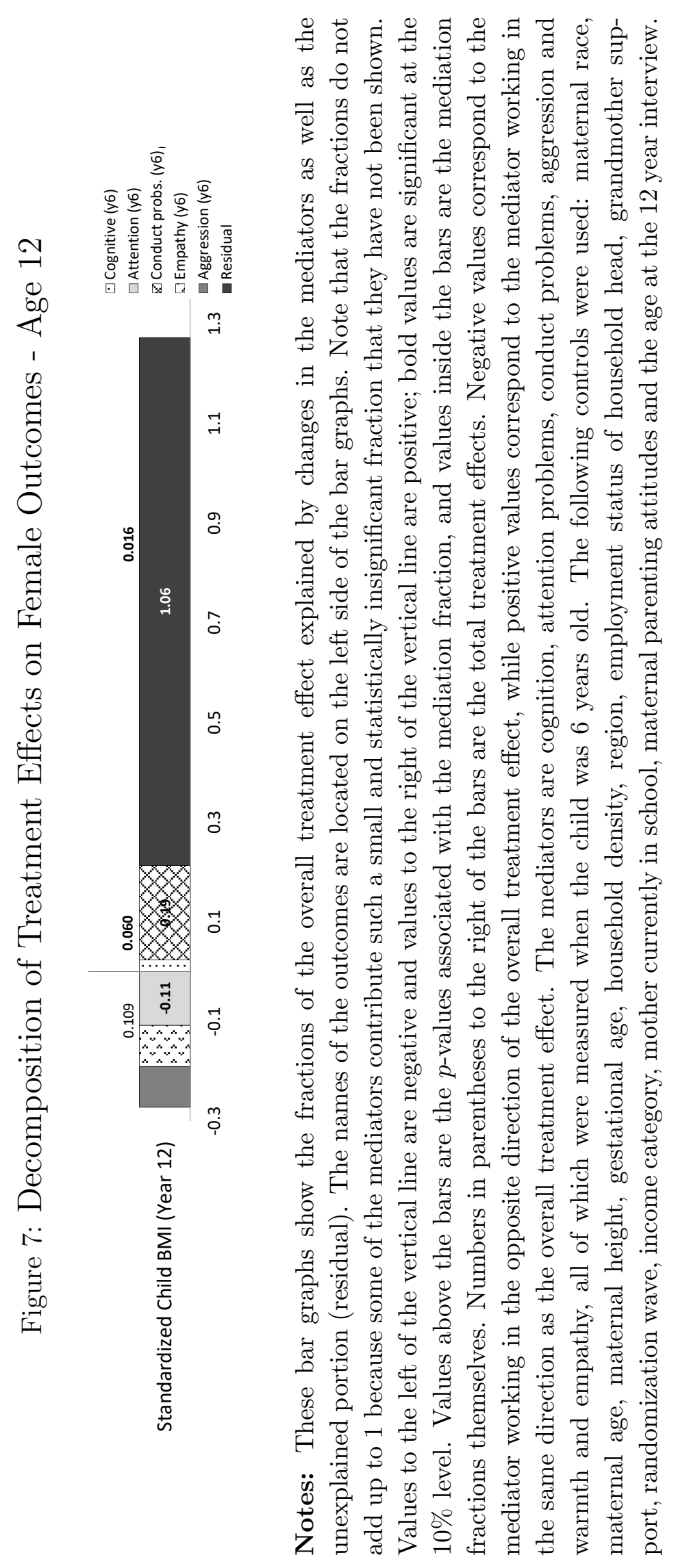




\section{Summary and Conclusion}

This paper analyzes the impacts through age 12 of the Nurse-Family Partnership Program as implemented in Memphis. The Nurse-Family Partnership (NFP) is the most cited home visiting program in the US (Howard and Brooks-Gunn, 2009a) and is one of the evidencebased home visiting models supported by the Maternal, Infant and Early Childhood Home Visiting (MEICHV) program. Currently, NFP surrogates have provided services for more than 200, 000 families in 43 states across the U.S.

We contribute to the existing literature in the NFP in several ways. We perform inference accounting for the special features of the NFP randomization protocol and addressing selective reporting of statistically significant outcomes (i.e., "cherry picking"). We show that most of the results of previous papers based on large sample asymptotic inference survive small sample permutation tests. However, fewer treatment effects survive corrections for multiple-hypothesis testing. Nonetheless, there are strong effects for boys that are sustained until age 12 .

In addition, this is the first paper that formally examines the mechanisms underlying NFP treatment effects. Following the methodology developed by Heckman et al. (2013), we decompose statistically significant treatment effects into interpretable components associated with NFP-induced changes in children's early skills and parental investments. We estimate the channels underlying treatment effects at age 6 (using maternal skills and investments effects at the end of the program as mediators ) and at age 12 (using the program effects at age 6 as mediators).

We find statistically significant treatment effects of NFP on birth weight for boys as well as improvements in the home environment, parenting attitudes, and maternal mental health for parents of both boys and girls at age $2 .{ }^{31}$ At age 6, the program improves cognitive skills for both boys and girls, while it enhances early socio-emotional skills for girls. These

\footnotetext{
${ }^{31}$ Our result of treatment effects on birth weight and other health at birth outcomes for boys contrasts with recent findings of no effects on similar outcomes in the Family Nurse Partnership program in the U.K. (Robling et al., 2016).
} 
treatment effects arise from program-induced improvements in maternal traits and early-life family investments at age 2 . The treatment effects for males persist through age 12 , but they do not persist for girls. Treated males outperform controls on a range of achievement scores. We find that $40 \%$ to $60 \%$ of male treatment effects at age 12 can be explained by enhanced cognitive skills measured at age 6 . The program has much weaker long-term effects for girls.

Our results are consistent with the evidence from well-known home visiting interventions like the Infant Health and Development Project (IHDP), and current nurse visitation programs like Preparing For Life (Ireland) (García et al., 2017a; Doyle, 2017; Besharov et al., 2011; Klebanov et al., 2001; Brooks-Gunn et al., 1994). Table 11 compares the results from the present study with the evidence from these two interventions.

Home visiting programs are widely advocated policy tools for promoting early child development by serving both disadvantaged parents and children at the same time. Several program models have existed since the 1960's, and the Nurse-Family Partnership is one of the most widely used and evaluated models. Overall, several home visiting programs have shown positive effects on improving parenting practices and the home environment, ${ }^{32}$ which are in line with our findings on those domains (Chen and Chan, 2016; Filene et al., 2013; Nievar et al., 2010). ${ }^{33}$ However, in a meta-analytic study of home visiting programs, Chen and Chan (2016) argue that few studies have looked at the effects of these programs on maternal mental health. We provide some evidence that the NFP improved maternal mastery and self-esteem and reduced maternal anxiety with effect sizes between 0.2-0.3 SD. Regarding child development outcomes, Filene et al. (2013) argue that previous home visiting interventions have shown mixed effects on child cognitive skills and the effect sizes vary significantly among the studies that have found effects (between 0.11-0.38 SDs). In this paper, we present evidence that the NFP had strong effects on cognitive skills at age six with effect sizes of 0.27 SDs for boys and 0.12 SDs for girls. These cognitive gains persist in program impacts on achievement

\footnotetext{
${ }^{32}$ For example, Filene et al. (2013) show that average effects sizes in home visiting evaluations range between 0.13 and 0.33 SDs.

${ }^{33}$ For reviews about home visiting program evidence see Chen and Chan (2016); Filene et al. (2013); Howard and Brooks-Gunn (2009b); Nievar et al. (2010); Paulsell et al. (2010); Sweet and Appelbaum (2004).
} 
test scores for boys at age 12. Filene et al. (2013) also demonstrate that the evidence of home visiting programs on birth outcomes is mixed. We find that the NFP improves these outcomes only for boys. Studies that review the evidence from home visiting programs argue that the existence of mixed effects across interventions can be explained because programs differ in the characteristics of the target population, goals, curriculum, services provided, frequency, the use of nurses versus paraprofessionals, and evaluation methods (Chen and Chan, 2016; Filene et al., 2013; Nievar et al., 2010; Paulsell et al., 2010).

This paper provides evidence that some of estimated NFP program effects for boys are sustained after the intervention ends. The more beneficial long-term effects for boys relative to girls is consistent with a large and growing body of evidence (Elango et al., 2014; García et al., 2017a,b and García et al., 2017). We also examine the underlying channels generating the estimated treatment effects and show that enhancements in the skills in early childhood and better parenting can explain a sizeable portion of NFP effects found in late childhood. 


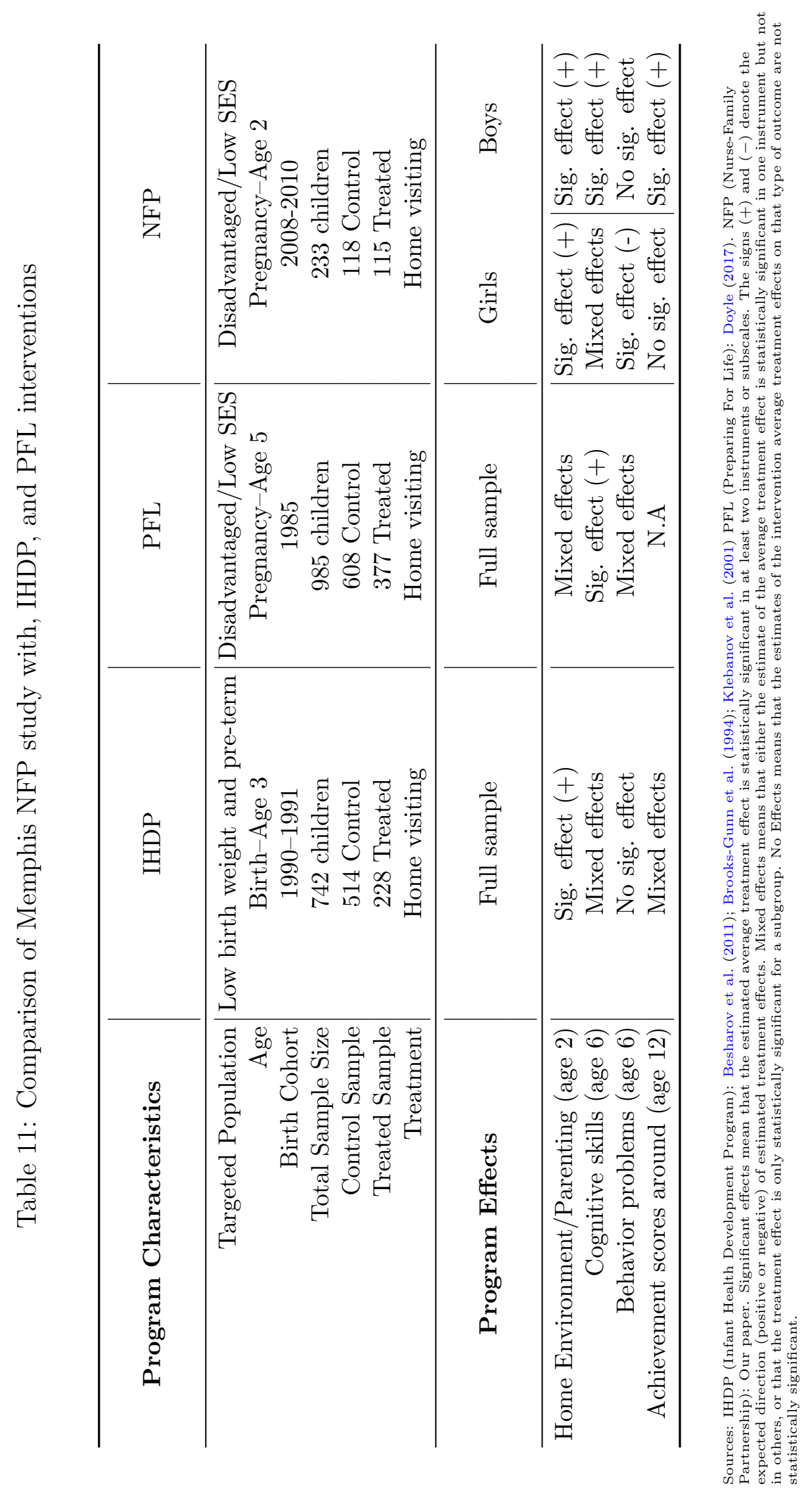




\section{References}

Almond, D. and J. Currie (2010). Human capital development before age five. Technical report, National Bureau of Economic Research.

Anderson, M. J. and P. Legendre (1999). An empirical comparison of permutation methods for tests of partial regression coefficients in a linear model. Journal of Statistical Computation and Simulation 62(3), 271-303.

Association, A. P. and A. P. A. T. F. on DSM-IV. (2000). Diagnostic and statistical manual of mental disorders: DSM-IV-TR. American Psychiatric Publishing, Inc.

Avellar, S., D. Paulsell, et al. (2011). Lessons learned from the home visiting evidence of effectiveness review. Technical report, Mathematica Policy Research.

Bartlett, M. S. (1937, July). The statistical conception of mental factors. British Journal of Psychology 28(1), 97-104.

Besharov, D., P. Germanis, C. Higney, and D. Call (2011). Infant health and development program. Assessments of twenty-six early childhood evaluations. Maryland School of Public Policy Welfare Reform Academy.

Breslau, N., J. DelDotto, G. Brown, S. Kumar, S. Ezhuthachan, K. Hufnagle, and E. Peterson (1994). A gradient relationship between low birth weight and iq at age 6 years. Archives of Pediatrics and Adolescent Medicine 148(4), 377.

Brooks-Gunn, J., C. M. McCarton, P. H. Casey, M. C. McCormick, C. R. Bauer, J. C. Bernbaum, J. Tyson, M. Swanson, F. C. Bennett, D. T. Scott, et al. (1994). Early intervention in low-birth-weight premature infants: Results through age 5 years from the infant health and development program. Jama 272(16), 1257-1262.

Burr, W. and C. Christensen (1992). Undesirable side effects of enhancing self-esteem. Family Relations, 460-464.

Chen, M. and K. L. Chan (2016). Effects of parenting programs on child maltreatment prevention: a meta-analysis. Trauma, Violence, 66 Abuse 17(1), 88-104.

Cunha, F. and J. J. Heckman (2007). Identifying and estimating the distributions of Ex Post and Ex Ante returns to schooling: A survey of recent developments. Labour Economics 14(6), 870-893.

Currie, J. and E. Moretti (2005). Biology as destiny? short and long-run determinants of intergenerational transmission of birth weight.

Currie, J. and M. Rossin-Slater (2015). Early-life origins of life-cycle well-being: Research and policy implications. Journal of Policy Analysis and Management 34(1), 208-242.

Daro, D. (2006). Home visitation assessing progress, managing expectations. Chapin Hall Center for Children. 
Doyle, O. (2017). The first 2,000 days and child skills: Evidence from a randomized experiment of home visiting.

Elango, S., , J. L. García, J. J. Heckman, and A. Hojman (2016). Early childhood education. In R. A. Moffitt (Ed.), Economics of Means-Tested Transfer Programs in the United States, Volume 2, Chapter 4, pp. 235-297. Chicago: University of Chicago Press.

Elango, S., A. Hojman, and S. Kuperman (2014). Perry cohort differences.

Filene, J. H., J. W. Kaminski, L. A. Valle, and P. Cachat (2013). Components associated with home visiting program outcomes: A meta-analysis. Pediatrics 132(Supplement 2), S100-S109.

Freedman, D. and D. Lane (1983, October). A nonstochastic interpretation of reported significance levels. Journal of Business and Economic Statistics 1(4), 292-298.

García, J. L., J. J. Heckman, D. E. Leaf, and M. J. Prados (2017a). The life-cycle benefits of an influential early childhood program. Unpublished.

García, J. L., J. J. Heckman, D. E. Leaf, and M. J. Prados (2017b). Quantifying the life-cycle benefits of a prototypical early childhood program. Unpublished.

García, J. L., J. J. Heckman, and A. L. Ziff (2017). Gender differences in the effects of early childhood education. Unpublished.

Gorsuch, R. (1983). Factor Analysis. Hillsdale, NJ: Lawrence Erlbaum Associates.

Heckman, J., R. Pinto, and P. Savelyev (2013). Understanding the mechanisms through which an influential early childhood program boosted adult outcomes. American Economic Review 103(6), 768-806.

Heckman, J. J. (2008). Role of income and family influence on child outcomes. Annals of the New York Academy of Sciences 1136(Reducing the Impact of Poverty on Health and Human Development: Scientific Approaches), 307-323.

Heckman, J. J., S. H. Moon, R. Pinto, P. A. Savelyev, and A. Q. Yavitz (2010, August). Analyzing social experiments as implemented: A reexamination of the evidence from the HighScope Perry Preschool Program. Quantitative Economics 1(1), 1-46.

Howard, K. and J. Brooks-Gunn (2009a). The role of home-visiting programs in preventing child abuse and neglect. The Future of Children, 119-146.

Howard, K. S. and J. Brooks-Gunn (2009b). The role of home-visiting programs in preventing child abuse and neglect. The Future of Children 19(2), 119-146.

Kitzman, H., D. Olds, R. Cole, C. Hanks, E. Anson, K. Arcoleo, D. Luckey, M. Knudtson, C. Henderson Jr, and J. Holmberg (2010). Enduring effects of prenatal and infancy home visiting by nurses on children: follow-up of a randomized trial among children at age 12 years. Archives of Pediatrics and Adolescent Medicine 164(5), 412. 
Kitzman, H., D. Olds, C. Henderson, C. Hanks, R. Cole, R. Tatelbaum, K. McConnochie, K. Sidora, D. Luckey, D. Shaver, et al. (1997). Effect of prenatal and infancy home visitation by nurses on pregnancy outcomes, childhood injuries, and repeated childbearing. Jama 278(8), 644-652.

Klebanov, P. K., J. Brooks-Gunn, and M. C. McCormick (2001). Maternal coping strategies and emotional distress: Results of an early intervention program for low birth weight young children. Developmental Psychology 37(5), 654.

Kraemer, S. (2000). The fragile male. Clinical Medicine NetPrints, 1.

Nelson, C. A. (2012, October). The effects of early life adversity on brain and behavioral development. Technical report, The Dana Foundation.

Nievar, M. A., L. A. Van Egeren, and S. Pollard (2010). A meta-analysis of home visiting programs: Moderators of improvements in maternal behavior. Infant mental health Journal 31(5), 499-520.

Olds, D. (2002). Prenatal and infancy home visiting by nurses: From randomized trials to community replication. Prevention Science 3(3), 153-172.

Olds, D., H. Kitzman, R. Cole, and J. Robinson (1997). Theoretical foundations of a program of home visitation for pregnant women and parents of young children. Journal of Community Psychology 25(1), 9-25.

Olds, D., H. Kitzman, R. Cole, J. Robinson, K. Sidora, D. Luckey, C. Henderson Jr, C. Hanks, J. Bondy, and J. Holmberg (2004). Effects of nurse home-visiting on maternal life course and child development: age 6 follow-up results of a randomized trial. Pediatrics 114(6), 1550.

Olds, D. L. (2012). Improving the life chances of vulnerable children and families with prenatal and infancy support of parents: the nurse-family partnership. Psychosocial Intervention 21(2), 129-143.

Paulsell, D., S. Avellar, E. S. Martin, P. Del Grosso, et al. (2010). Home visiting evidence of effectiveness review: Executive summary. Technical report, Mathematica Policy Research.

Pearlin, L. I. and C. Schooler (1978). The structure of coping. Journal of health and social behavior, 2-21.

Robins, J. M., A. Rotnitzky, and L. P. Zhao (1994). Estimation of regression coefficients when some regressors are not always observed. Journal of the American Statistical Association 89(427), 846-866.

Robling, M., M.-J. Bekkers, K. Bell, C. C. Butler, R. Cannings-John, S. Channon, B. C. Martin, J. W. Gregory, K. Hood, A. Kemp, J. Kenkre, A. A. Montgomery, G. Moody, E. Jones-Owen, K. Pickett, G. Richardson, Z. E. S. Roberts, S. Ronaldson, J. Sanders, E. Stamuli, and D. Torgerson (2016, January). Effectiveness of a nurse-led intensive home-visitation programme for first-time teenage mothers (Building Blocks): A pragmatic randomised controlled trial. The Lancet 387(10014, 9-15), 146-155. 
Romano, J. P. and M. Wolf (2005, March). Exact and approximate stepdown methods for multiple hypothesis testing. Journal of the American Statistical Association 100(469), 94-108.

Rosenberg, M. (1965). Society and the adolescent self-image, Volume 11. Princeton university press Princeton, NJ.

Sandner, M., T. Cornelissen, T. Jungmann, and P. Herrmann (2017). Hceo working paper series.

Schore, A. N. (2017). All our sons: The developmental neurobiology and neuroendocrinology of boys at risk. Infant Mental Health Journal 38(1), 15-52.

Sweet, M. A. and M. I. Appelbaum (2004). Is home visiting an effective strategy? a metaanalytic review of home visiting programs for families with young children. Child development 75(5), 1435-1456.

VanderWeele, T. (2015). Explanation in Causal Inference: Methods for Mediation and Interaction. Oxford University Press.

Veit, C. T. and J. E. Ware (1983). The structure of psychological distress and well-being in general populations. Journal of consulting and clinical psychology 51(5), 730. 